\title{
Dynamical Analysis in a Delayed Predator-Prey System with Stage-Structure for Both the Predator and the Prey
}

\author{
Zizhen Zhang ${ }^{1,2}$ and Huizhong Yang ${ }^{1}$ \\ ${ }^{1}$ Key Laboratory of Advanced Process Control for Light Industry (Ministry of Education), Jiangnan University, Wuxi 214122, China \\ ${ }^{2}$ School of Management Science and Engineering, Anhui University of Finance and Economics, Bengbu 233030, China \\ Correspondence should be addressed to Huizhong Yang; yhz@jiangnan.edu.cn
}

Received 24 June 2013; Accepted 27 November 2013; Published 19 February 2014

Academic Editor: Zhengqiu Zhang

Copyright (c) 2014 Z. Zhang and H. Yang. This is an open access article distributed under the Creative Commons Attribution License, which permits unrestricted use, distribution, and reproduction in any medium, provided the original work is properly cited.

A predator-prey system with two delays and stage-structure for both the predator and the prey is considered. Sufficient conditions for the local stability and the existence of periodic solutions via Hopf bifurcation with respect to both delays are obtained by analyzing the distribution of the roots of the associated characteristic equation. Specially, the direction of the Hopf bifurcation and the stability of the periodic solutions bifurcating from the Hopf bifurcation are determined by applying the normal form theory and center manifold argument. Some numerical simulations for justifying the theoretical analysis are also provided.

\section{Introduction}

The predator-prey systems with stage-structure have been studied by many authors [1-5]. In [2], Aiello and Freedman built a stage-structured model of single species:

$$
\begin{gathered}
\frac{d x_{i}(t)}{d t}=\alpha x_{m}(t)-\gamma x_{i}(t)-\alpha e^{-\gamma \tau} x_{m}(t-\tau), \\
\frac{d x_{m}(t)}{d t}=\alpha e^{-\gamma \tau} x_{m}(t-\tau)-\beta x_{m}^{2}(t),
\end{gathered}
$$

where $x_{i}(t)$ and $x_{m}(t)$ denote the densities of the immature population and the mature population at time $t$, respectively. However, it is well known that the predator-prey relationship often appears in population ecology. Multispecies predatorprey systems with stage structure are very important and have received much attention in recent years [6-16].

In [13], Xu investigated a predator-prey system with stage structure for the predator:

$$
\frac{d x(t)}{d t}=x(t)\left(r-a x(t)-\frac{a_{1} y_{2}(t)}{1+m x(t)}\right),
$$

$$
\begin{gathered}
\frac{d y_{1}(t)}{d t}=\frac{a_{2} x(t-\tau) y_{2}(t-\tau)}{1+m x(t-\tau)}-r_{1} y_{1}(t)-D y_{1}(t), \\
\frac{d y_{2}(t)}{d t}=D y_{1}(t)-r_{2} y_{2}(t),
\end{gathered}
$$

where $x(t)$ denotes the density of the prey at time t. $y_{1}(t)$ and $y_{2}(t)$ denote the densities of the immature predator and the mature predator at time $t$, respectively. $\mathrm{Xu}$ [13] proved that system (2) is permanent under certain conditions by means of the persistence theory on infinite-dimensional systems. And sufficient conditions were derived for the local and global stability of the coexistence equilibrium of the system. In [15], F. Li and $\mathrm{H}$. Li investigated a predator-prey system with stage structure for the prey:

$$
\begin{gathered}
\frac{d x_{1}(t)}{d t}=a x_{2}(t)-r_{1} x_{1}(t)-b x_{1}(t), \\
\frac{d x_{2}(t)}{d t}=b x_{1}(t)-r_{2} x_{2}(t)-b_{1} x_{2}^{2}(t)-\frac{a_{1} x_{2}^{2}(t) y(t)}{1+m x_{2}^{2}(t)} \\
\frac{d y(t)}{d t}=\frac{a_{2} x_{2}^{2}(t-\tau) y(t-\tau)}{1+m x_{2}^{2}(t-\tau)}-r y(t),
\end{gathered}
$$


where $x_{1}(t)$ and $x_{2}(t)$ denote the densities of the immature prey and the mature prey at time $t$, respectively. $y(t)$ denotes the density of the predator at time $t$. F. Li and H. Li [15] studied the effect of the gestation time of the predator on the dynamics of system (3).

Since both the predator and the prey have a life history that takes them through immature stage and mature stage, it is reasonable to consider the predator-prey system with stagestructure for both the predator and the prey. Starting from this point, Wang [17] proposed the following delayed system:

$$
\begin{gathered}
\frac{d x_{1}(t)}{d t}=r x_{2}(t)-a x_{1}^{2}(t)-\left(d_{1}+r_{1}\right) x_{1}(t)-a_{1} x_{1}(t) y_{2}(t), \\
\frac{d x_{2}(t)}{d t}=r_{1} x_{1}(t)-d_{2} x_{2}(t) \\
\frac{d y_{1}(t)}{d t}=a_{2} x_{1}(t-\tau) y_{2}(t-\tau)-\left(d_{3}+r_{2}\right) y_{1}(t) \\
\frac{d y_{2}(t)}{d t}=r_{2} y_{1}(t)-d_{4} y_{2}(t)
\end{gathered}
$$

where $x_{1}(t)$ and $x_{2}(t)$ denote the densities of the immature prey and the mature prey at time $t$, respectively. $y_{1}(t)$ and $y_{2}(t)$ denote the densities of the immature predator and the mature predator at time $t$, respectively. $a$ is the intraspecific competition coefficient of the immature prey. $a_{1}$ and $a_{2}$ are the interspecific interaction coefficients between the immature prey and the mature predator. $d_{1}$ and $d_{2}$ are the death rates of the immature prey and the mature prey, respectively. $d_{3}$ and $d_{4}$ are the death rates of the immature predator and the mature predator, respectively. $r$ is the birth rate of the immature prey. $r_{1}$ and $r_{2}$ are the transformation rates from immature individuals to mature individuals for the prey and the predator, respectively. All the parameters in system (4) are assumed to be positive constants. And $\tau \geq 0$ is a constant delay due to the gestation of the mature predator. Wang [17] considered the bifurcation phenomenon and the properties of periodic solutions of system (4).

The predator-prey systems with single delay have been investigated by many researchers. There are also many papers on the bifurcations of predator-prey systems with two or multiple delays [18-24]. Cui and Yan [20] investigated the stability and Hopf bifurcations of a three-species LotkaVolterra food chain system by taking the sum of the two delays in the system as the bifurcation parameter. Gakkhar and Singh [23] investigated a modified Leslie-Gower predatorprey system with two delays and obtained the sufficient conditions for existence of Hopf bifurcation for possible combinations of the two delays. Motivated by the work above, we consider the following predator-prey system with two delays:

$$
\begin{gathered}
\frac{d x_{1}(t)}{d t}=r x_{2}(t)-a x_{1}(t) x_{1}\left(t-\tau_{1}\right) \\
-\left(d_{1}+r_{1}\right) x_{1}(t)-a_{1} x_{1}(t) y_{2}(t), \\
\frac{d x_{2}(t)}{d t}=r_{1} x_{1}(t)-d_{2} x_{2}(t)
\end{gathered}
$$

$$
\begin{gathered}
\frac{d y_{1}(t)}{d t}=a_{2} x_{1}\left(t-\tau_{2}\right) y_{2}\left(t-\tau_{2}\right)-\left(d_{3}+r_{2}\right) y_{1}(t), \\
\frac{d y_{2}(t)}{d t}=r_{2} y_{1}(t)-d_{4} y_{2}(t),
\end{gathered}
$$

where $\tau_{1} \geq 0$ is feedback delay of the immature prey to the growth of the species itself and $\tau_{2} \geq 0$ is the time delay due to the gestation of the mature predator.

The rest of this paper is organized as follows. In Section 2, sufficient conditions are established for the local stability of the positive equilibrium and the existence of Hopf bifurcation for possible combinations of the two delays in system (5). Section 3 is devoted to the properties of the Hopf bifurcation on the normal form theory and center manifold argument. Numerical simulations supporting the theoretical analysis are presented in Section 4. Finally, conclusions are given in Section 5.

\section{Local Stability and Existence of Hopf Bifurcation}

Considering the significance of ecology, we are interested only in positive equilibrium of system (5). It is not difficult to verify that if the condition $(H)$ :

$$
a_{2} r r_{1} r_{2}>a d_{2} d_{4}\left(d_{3}+r_{2}\right)+a_{2} d_{2} r_{2}\left(d_{1}+r_{1}\right)
$$

holds, then system (5) has a unique positive equilibrium $E^{*}\left(x_{1}^{*}, x_{2}^{*}, y_{1}^{*}, y_{2}^{*}\right)$, where

$$
\begin{gathered}
x_{1}^{*}=\frac{d_{4}\left(d_{3}+r_{2}\right)}{a_{2} r_{2}}, \quad x_{2}^{*}=\frac{d_{4} r_{1}\left(d_{3}+r_{2}\right)}{a_{2} d_{2} r_{2}}, \\
y_{1}^{*}=\frac{d_{4}}{r_{2}} y_{2}^{*}, \quad y_{2}^{*}=\frac{r r_{1}-d_{2}\left(d_{1}+r_{1}\right)}{a_{1} d_{2}}-\frac{a d_{4}\left(d_{3}+r_{2}\right)}{a_{1} a_{2} r_{2}} .
\end{gathered}
$$

Let $\bar{x}_{1}=x_{1}-x_{1}^{*}, \bar{x}_{2}=x_{2}-x_{2}^{*}, \bar{y}_{1}=y_{1}-y_{1}^{*}$, and $\bar{y}_{2}=$ $y_{2}-y_{2}^{*}$. Dropping the bars, system (5) can be transformed to the following form:

$$
\begin{aligned}
& \frac{d x_{1}(t)}{d t}=a_{11} x_{1}(t)+a_{12} x_{2}(t)+a_{14} y_{2}(t)+b_{11} x_{1}\left(t-\tau_{1}\right) \\
&+g_{1} x_{1}(t) y_{2}(t)+g_{2} x_{1}(t) x_{1}\left(t-\tau_{1}\right), \\
& \frac{d x_{2}(t)}{d t}=a_{21} x_{1}(t)+a_{22} x_{2}(t), \\
& \frac{d y_{1}(t)}{d t}= a_{33} y_{1}(t)+c_{31} x_{1}\left(t-\tau_{2}\right)+c_{34} y_{2}\left(t-\tau_{2}\right) \\
&+h_{1} x_{1}\left(t-\tau_{2}\right) y_{2}\left(t-\tau_{2}\right), \\
& \frac{d y_{2}(t)}{d t}=a_{43} y_{1}(t)+a_{44} y_{2}(t),
\end{aligned}
$$


where

$$
\begin{gathered}
a_{11}=-a x_{1}^{*}-a_{1} y_{2}^{*}-\left(d_{1}+r_{1}\right), \\
a_{12}=r, \quad a_{14}=-a_{1} x_{1}^{*}, \\
a_{21}=r_{1}, \quad a_{22}=-d_{2}, \quad a_{33}=-\left(d_{3}+r_{2}\right), \\
a_{43}=r_{2}, \quad a_{44}=-d_{4}, \quad b_{11}=-a x_{1}^{*}, \\
c_{31}=a_{2} y_{2}^{*}, \quad c_{34}=a_{2} x_{1}^{*}, \\
g_{1}=-a_{1}, \quad g_{2}=-a, \quad h_{1}=a_{2} .
\end{gathered}
$$

The linear system of system (8) is

$$
\begin{gathered}
\frac{d x_{1}(t)}{d t}=a_{11} x_{1}(t)+a_{12} x_{2}(t)+a_{14} y_{2}(t)+b_{11} x_{1}\left(t-\tau_{1}\right), \\
\frac{d x_{2}(t)}{d t}=a_{21} x_{1}(t)+a_{22} x_{2}(t), \\
\frac{d y_{1}(t)}{d t}=a_{33} y_{1}(t)+c_{31} x_{1}\left(t-\tau_{2}\right)+c_{34} y_{2}\left(t-\tau_{2}\right), \\
\frac{d y_{2}(t)}{d t}=a_{43} y_{1}(t)+a_{44} y_{2}(t) .
\end{gathered}
$$

The characteristic equation of system (10) is as follows:

$$
\begin{aligned}
\lambda^{4}+m_{3} \lambda^{3}+m_{2} \lambda^{2}+m_{1} \lambda+m_{0} \\
+\left(n_{3} \lambda^{3}+n_{2} \lambda^{2}+n_{1} \lambda+n_{0}\right) e^{-\lambda \tau_{1}} \\
+\left(p_{2} \lambda^{2}+p_{1} \lambda+p_{0}\right) e^{-\lambda \tau_{2}} \\
+\left(q_{1} \lambda+q_{0}\right) e^{-\lambda\left(\tau_{1}+\tau_{2}\right)}=0,
\end{aligned}
$$

where

$$
\begin{gathered}
m_{3}=-\left(a_{11}+a_{22}+a_{33}+a_{44}\right), \\
m_{2}=a_{11} a_{22}+a_{33} a_{44}-a_{12} a_{21} \\
+\left(a_{11}+a_{22}\right)\left(a_{33}+a_{44}\right), \\
m_{1}=\left(a_{12} a_{21}-a_{11} a_{22}\right)\left(a_{33}+a_{44}\right) \\
-\left(a_{11}+a_{22}\right) a_{33} a_{44}, \\
m_{0}=\left(a_{11} a_{22}-a_{12} a_{21}\right) a_{33} a_{44}, \\
n_{3}=-b_{11}, \quad n_{2}=\left(a_{22}+a_{33}+a_{44}\right) b_{11}, \\
n_{1}=-a_{22} b_{11}\left(a_{33}+a_{44}\right)-a_{33} a_{44} b_{11}, \\
n_{0}=a_{22} a_{33} a_{44} b_{11}, \quad p_{2}=-a_{43} c_{34}, \\
p_{1}=\left(a_{11}+a_{22}\right) a_{43} c_{34}-a_{14} a_{43} c_{31}, \\
p_{0}=\left(a_{12} a_{21}-a_{11} a_{22}\right) a_{43} c_{34}+a_{14} a_{22} a_{43} c_{31}, \\
q_{1}=a_{43} b_{11} c_{34}, \quad q_{0}=-a_{22} a_{43} b_{11} c_{34} .
\end{gathered}
$$

Case $1\left(\tau_{1}=\tau_{2}=0\right)$. For $\tau_{1}=\tau_{2}=0$, (11) can be rewritten in the following form:

$$
\lambda^{4}+A_{13} \lambda^{3}+A_{12} \lambda^{2}+A_{11} \lambda+A_{10}=0,
$$

where

$$
\begin{gathered}
A_{13}=m_{3}+n_{3} \\
=2 a x_{1}^{*}+a_{1} y_{2}^{*}+d_{1}+d_{2}+d_{3}+d_{4}+r_{1}+r_{2}, \\
A_{12}=m_{2}+n_{2}+p_{2}, \\
A_{11}=m_{1}+n_{1}+p_{1}+q_{1}, \quad A_{10}=m_{0}+n_{0}+p_{0}+q_{0} .
\end{gathered}
$$

Clearly, $A_{13}>0$. Therefore, by the Routh-Hurwitz theorem, if the conditions $\left(H_{11}\right) A_{10}>0, A_{13} A_{12}>A_{11}$ and $\left(H_{12}\right) A_{11} A_{12} A_{13}>A_{11}^{2}+A_{10} A_{13}^{2}$ hold, then the positive equilibrium $E^{*}\left(x_{1}^{*}, x_{2}^{*}, y_{1}^{*}, y_{2}^{*}\right)$ of system (5) without time delay is locally asymptotically stable.

Case $2\left(\tau_{1}>0, \tau_{2}=0\right)$. On substituting $\tau_{2}=0$, (11) becomes

$$
\begin{aligned}
& \lambda^{4}+A_{23} \lambda^{3}+A_{22} \lambda^{2}+A_{21} \lambda+A_{20} \\
& +\left(B_{23} \lambda^{3}+B_{22} \lambda^{2}+B_{21} \lambda+B_{20}\right) e^{-\lambda \tau_{1}}=0,
\end{aligned}
$$

where

$$
\begin{aligned}
A_{23}=m_{3}, & A_{22}=m_{2}+p_{2}, \\
A_{21}=m_{1}+p_{1}, & A_{20}=m_{0}+p_{0}, \\
B_{23}=n_{3}, & B_{22}=n_{2}, \\
B_{21}=n_{1}+q_{1}, & B_{20}=n_{0}+q_{0} .
\end{aligned}
$$

Let $\lambda=i \omega_{1}\left(\omega_{1}>0\right)$ be the root of (15). Then, we have

$$
\begin{gathered}
\left(B_{21} \omega_{1}-B_{23} \omega_{1}^{3}\right) \sin \tau_{1} \omega_{1}+\left(B_{20}-B_{22} \omega_{1}^{2}\right) \cos \tau_{1} \omega_{1} \\
=A_{22} \omega_{1}^{2}-\omega_{1}^{4}-A_{20}, \\
\left(B_{21} \omega_{1}-B_{23} \omega_{1}^{3}\right) \cos \tau_{1} \omega_{1}-\left(B_{20}-B_{22} \omega_{1}^{2}\right) \sin \tau_{1} \omega_{1} \\
=A_{23} \omega_{1}^{3}-A_{21} \omega_{1},
\end{gathered}
$$

which follows that

$$
\omega_{1}^{8}+e_{23} \omega_{1}^{6}+e_{22} \omega_{1}^{4}+e_{21} \omega_{1}+e_{20}=0
$$

with

$$
\begin{gathered}
e_{23}=A_{23}^{2}-B_{23}^{2}-2 A_{22}, \\
e_{22}=A_{22}^{2}-B_{22}^{2}+2 B_{21} B_{23}-2 A_{21} A_{23}+2 A_{20}, \\
e_{21}=A_{21}^{2}-B_{21}^{2}-2 A_{20} A_{22}+2 B_{20} B_{22}, \\
e_{20}=A_{20}^{2}-B_{20}^{2} .
\end{gathered}
$$

Let $\omega_{1}^{2}=v_{1}$, then (18) becomes

$$
v_{1}^{4}+e_{23} v_{1}^{3}+e_{22} v_{1}^{2}+e_{21} v_{1}+e_{20}=0 .
$$


Define

$$
\begin{gathered}
f_{1}\left(v_{1}\right)=v_{1}^{4}+e_{23} v_{1}^{3}+e_{22} v_{1}^{2}+e_{21} v_{1}+e_{20}, \\
p=\frac{1}{2} e_{22}-\frac{3}{16} e_{23}^{2}, \quad q=\frac{1}{32} e_{23}^{3}-\frac{1}{8} e_{22} e_{23}+e_{21}, \\
\alpha_{1}=\left(\frac{q}{2}\right)^{3}+\left(\frac{p}{3}\right)^{3}, \quad \beta_{1}=-\frac{1}{2}+\frac{\sqrt{3}}{2} i \\
y_{1}=\sqrt[3]{-\frac{q}{2}+\sqrt{\alpha_{1}}}+\sqrt[3]{-\frac{q}{2}-\sqrt{\alpha_{1}}}, \\
y_{2}=\beta_{1} \sqrt[3]{-\frac{q}{2}+\sqrt{\alpha_{1}}}+\beta_{1}^{2} \sqrt[3]{-\frac{q}{2}-\sqrt{\alpha_{1}}}, \\
y_{3}=\beta_{1}^{2} \sqrt[3]{-\frac{q}{2}+\sqrt{\alpha_{1}}}+\beta_{1} \sqrt[3]{-\frac{q}{2}-\sqrt{\alpha_{1}}} \\
v_{1 i}=y_{i}-\frac{3 e_{23}}{4}, \quad i=1,2,3 .
\end{gathered}
$$

Discussion about the roots of (20) is similar to that in [25]. Therefore, we have the following lemma.

Lemma 1. For (20), one has the following.

(i) If $e_{20}<0,(20)$ has at least one positive root.

(ii) If $e_{20} \geq 0$ and $\alpha_{1} \geq 0,(20)$ has positive roots if and only if $v_{11}>0$ and $f_{1}\left(v_{11}\right)<0$.

(iii) If $e_{20} \geq 0$ and $\alpha_{1}<0$, (20) has positive roots if and only if there exists at least one $v_{1 *} \in\left\{v_{11}, v_{12}, v_{13}\right\}$ such that $v_{1 *}>0$ and $f_{1}\left(v_{1 *}\right) \leq 0$.

In what follows, one assumes that $\left(H_{21}\right)$ : the coefficients in $f_{1}\left(v_{1}\right)$ satisfy one of the following conditions in $(a)-(c)$ :

(a) $e_{20}<0$;

(b) $e_{20} \geq 0, \alpha_{1} \geq 0, v_{11}>0$ and $f_{1}\left(v_{11}\right)<0$;

(c) $e_{20} \geq 0, \alpha_{1}<0$, and there exists at least one $v_{1 *} \in$ $\left\{v_{11}, v_{12}, v_{13}\right\}$ such that $v_{1 *}>0$ and $f_{1}\left(v_{1 *}\right) \leq 0$.

If $\left(\mathrm{H}_{21}\right)$ holds, one knows that (18) has at least a positive root $\omega_{10}$ such that (15) has a pair of purely imaginary roots $\pm i \omega_{10}$. The corresponding critical value of the delay is

$$
\begin{gathered}
\tau_{1 k}=\frac{1}{\omega_{10}} \\
\times \arccos \left\{\left(\left(B_{22}-A_{23} B_{23}\right) \omega_{10}^{6}\right.\right. \\
+\left(A_{21} B_{23}+A_{23} B_{21}\right. \\
\left.\left.\quad-A_{22} B_{22}-B_{20}\right) \omega_{10}^{4}\right) \\
\times\left(B_{23}^{2} \omega_{10}^{6}+\left(B_{22}^{2}-2 B_{21} B_{23}\right) \omega_{10}^{4}\right. \\
\left.\quad+\left(B_{21}^{2}-2 B_{20} B_{22}\right) \omega_{10}^{2}+B_{20}^{2}\right)^{-1} \\
+\left(\left(A_{20} B_{22}+A_{22} B_{20}-A_{21} B_{21}\right) \omega_{10}^{2}\right. \\
\left.\quad-A_{20} B_{20}\right)
\end{gathered}
$$

$$
\begin{aligned}
\times\left(B_{23}^{2} \omega_{10}^{6}+\left(B_{22}^{2}-2 B_{21} B_{23}\right) \omega_{10}^{4}\right. \\
\left.\left.+\left(B_{21}^{2}-2 B_{20} B_{22}\right) \omega_{10}^{2}+B_{20}^{2}\right)^{-1}\right\}
\end{aligned}
$$

$$
+\frac{2 k \pi}{\omega_{10}}, \quad k=0,1,2, \ldots
$$

Differentiating both sides of (15) regarding $\tau_{1}$, one gets

$$
\begin{aligned}
{\left[\frac{d \lambda}{d \tau_{1}}\right]^{-1}=} & -\frac{4 \lambda^{3}+3 A_{23} \lambda^{2}+2 A_{22} \lambda+A_{21}}{\lambda\left(\lambda^{4}+A_{23} \lambda^{3}+A_{22} \lambda^{2}+A_{21} \lambda+A_{20}\right)} \\
& +\frac{3 B_{23} \lambda^{2}+2 B_{22} \lambda+B_{21}}{\lambda\left(B_{23} \lambda^{3}+B_{22} \lambda^{2}+B_{21} \lambda+B_{20}\right)}-\frac{\tau_{1}}{\lambda} .
\end{aligned}
$$

Thus,

$$
\begin{aligned}
\operatorname{Re}\left[\frac{d \lambda}{d \tau_{1}}\right]_{\tau_{1}=\tau_{10}}^{-1} & \\
=f_{1}^{\prime}\left(v_{1}^{*}\right) \times( & B_{23}^{2} \omega_{10}^{6}+\left(B_{22}^{2}-2 B_{21} B_{23}\right) \omega_{10}^{4} \\
& \left.+\left(B_{21}^{2}-2 B_{20} B_{22}\right) \omega_{10}^{2}+B_{20}^{2}\right)^{-1},
\end{aligned}
$$

where $v_{1}^{*}=\omega_{10}^{2}$. Therefore, if the condition $\left(H_{22}\right): f_{1}^{\prime}\left(v_{1}^{*}\right) \neq 0$ holds, then $\operatorname{Re}\left[d \lambda / d \tau_{1}\right]_{\tau_{1}=\tau_{10}}^{-1} \neq 0$. Namely, if the condition $\left(H_{22}\right): f_{1}^{\prime}\left(v_{1}^{*}\right) \neq 0$ holds, the transversality condition is satisfied. By the Hopf bifurcation theorem in [26], one has the following results.

Theorem 2. If the conditions $\left(H_{21}\right)-\left(H_{22}\right)$ hold, then

(i) the positive equilibrium $E^{*}\left(x_{1}^{*}, x_{2}^{*}, y_{1}^{*}, y_{2}^{*}\right)$ of system (5) is asymptotically stable for $\tau_{1} \in\left[0, \tau_{10}\right)$;

(ii) system (5) undergoes a Hopf bifurcation at the equilibrium $E^{*}\left(x_{1}^{*}, x_{2}^{*}, y_{1}^{*}, y_{2}^{*}\right)$ when $\tau_{1}=\tau_{10}$, and a family of periodic solutions bifurcate from $E^{*}\left(x_{1}^{*}, x_{2}^{*}, y_{1}^{*}, y_{2}^{*}\right)$ near $\tau_{1}=\tau_{10}$.

Case $3\left(\tau_{1}=0, \tau_{2}>0\right)$. On substituting $\tau_{1}=0$, (11) becomes

$$
\begin{aligned}
\lambda^{4}+A_{33} \lambda^{3}+A_{32} \lambda^{2}+A_{31} \lambda+A_{30} & \\
& +\left(B_{32} \lambda^{2}+B_{31} \lambda+B_{30}\right) e^{-\lambda \tau_{2}}=0
\end{aligned}
$$

where

$$
\begin{gathered}
A_{33}=m_{3}+n_{3}, \quad A_{32}=m_{2}+n_{2}, \\
A_{31}=m_{1}+n_{1}, \quad A_{30}=m_{0}+n_{0}, \\
B_{32}=p_{2}, \quad B_{31}=p_{1}+q_{1}, \quad B_{30}=p_{0}+q_{0} .
\end{gathered}
$$

Let $\lambda=i \omega_{2}\left(\omega_{2}>0\right)$ be the root of (25). Then, we have

$$
\begin{gathered}
B_{31} \sin \tau_{2} \omega_{2}+\left(B_{30}-B_{32} \omega_{2}^{2}\right) \cos \tau_{2} \omega_{2} \\
=A_{32} \omega_{2}^{2}-\omega_{2}^{4}-A_{30}, \\
B_{31} \cos \tau_{2} \omega_{2}-\left(B_{30}-B_{32} \omega_{2}^{2}\right) \sin \tau_{2} \omega_{2} \\
=A_{33} \omega_{2}^{3}-A_{31} \omega_{2} .
\end{gathered}
$$


It follows that

$$
\omega_{2}^{8}+e_{33} \omega_{2}^{6}+e_{32} \omega_{2}^{4}+e_{31} \omega_{2}+e_{30}=0
$$

where

$$
\begin{gathered}
e_{33}=A_{33}^{2}-2 A_{32}, \\
e_{32}=A_{32}^{2}-B_{32}^{2}+2 A_{30}-2 A_{31} A_{33}, \\
e_{31}=A_{31}^{2}-B_{31}^{2}-2 A_{30} A_{32}+2 B_{30} B_{32}, \\
e_{30}=A_{30}^{2}-B_{30}^{2} .
\end{gathered}
$$

Let $\omega_{2}^{2}=v_{2}$, then (28) becomes

$$
v_{2}^{4}+e_{33} v_{2}^{3}+e_{32} v_{2}^{2}+e_{31} v_{2}+e_{30}=0
$$

Define

$$
\begin{gathered}
f_{2}\left(v_{2}\right)=v_{2}^{4}+e_{33} v_{2}^{3}+e_{32} v_{2}^{2}+e_{31} v_{2}+e_{30} \\
m=\frac{1}{2} e_{32}-\frac{3}{16} e_{33}^{2}, \quad n=\frac{1}{32} e_{33}^{3}-\frac{1}{8} e_{32} e_{33}+e_{31}, \\
\alpha_{2}=\left(\frac{n}{2}\right)^{3}+\left(\frac{m}{3}\right)^{3}, \quad \beta_{2}=-\frac{1}{2}+\frac{\sqrt{3}}{2} i \\
z_{1}=\sqrt[3]{-\frac{n}{2}+\sqrt{\alpha_{2}}}+\sqrt[3]{-\frac{n}{2}-\sqrt{\alpha_{2}}} \\
z_{2}=\beta_{2} \sqrt[3]{-\frac{n}{2}+\sqrt{\alpha_{2}}}+\beta_{2}^{2} \sqrt[3]{-\frac{n}{2}-\sqrt{\alpha_{2}}} \\
z_{3}=\beta_{2}^{2} \sqrt[3]{-\frac{n}{2}+\sqrt{\alpha_{2}}}+\beta_{2} \sqrt[3]{-\frac{n}{2}-\sqrt{\alpha_{2}}} \\
v_{2 i}=z_{i}-\frac{3 e_{33}}{4}, \quad i=1,2,3 .
\end{gathered}
$$

According to Lemma 1, we know that if $\left(H_{31}\right)$ the coefficients in $f_{2}\left(v_{2}\right)$ satisfy one of the following conditions in $\left(\mathrm{a}^{\prime}\right)-\left(\mathrm{c}^{\prime}\right)$ :

$\left(\mathrm{a}^{\prime}\right) e_{30}<0$

$\left(\mathrm{b}^{\prime}\right) e_{30} \geq 0, \alpha_{2} \geq 0, v_{21}>0$, and $f_{2}\left(v_{21}\right)<0$;

$\left(c^{\prime}\right) e_{30} \geq 0, \alpha_{2}<0$, and there exists at least one $v_{2 *} \epsilon$ $\left\{v_{21}, v_{22}, v_{23}\right\}$ such that $v_{2 *}>0$ and $f_{2}\left(v_{2 *}\right) \leq 0$.

Then, (28) has at least a positive roots $\omega_{20}$ such that (25) has a pair of purely imaginary roots $\pm i \omega_{20}$. The corresponding critical value of the delay is

$$
\begin{array}{r}
\tau_{2 k}=\frac{1}{\omega_{20}}<\arccos \left(\left(B_{32} \omega_{20}^{6}+\left(A_{33} B_{31}-A_{32} B_{32}-B_{30}\right) \omega_{20}^{4}\right.\right. \\
\left.+\left(A_{30} B_{32}+A_{32} B_{30}-A_{31} B_{31}\right) \omega_{20}^{2}\right)
\end{array}
$$

$$
\begin{aligned}
& \times\left(B_{32}^{2} \omega_{20}^{4}+\left(B_{31}^{2}-2 B_{30} B_{32}\right)\right. \\
& \left.\left.\times \omega_{20}^{2}+B_{30}^{2}\right)^{-1}\right)
\end{aligned}
$$$$
+\frac{2 k \pi}{\omega_{20}}, \quad k=0,1,2, \ldots
$$

Similarly as in Case 2, we can conclude that if the condition $\left(H_{32}\right) f_{2}^{\prime}\left(v_{2}^{*}\right) \neq 0,\left(v_{2}^{*}=\omega_{20}^{2}\right)$, then $\operatorname{Re}\left[d \lambda / d \tau_{2}\right]_{\tau_{2}=\tau_{20}}^{-1} \neq 0$. That is, if the condition $\left(H_{32}\right) f_{2}^{\prime}\left(v_{2}^{*}\right) \neq 0$ holds, the transversality condition is satisfied. According to the Hopf bifurcation theorem in [26], we obtain the following results.

Theorem 3. If the conditions $\left(H_{31}\right)-\left(H_{32}\right)$ hold, then

(i) the positive equilibrium $E^{*}\left(x_{1}^{*}, x_{2}^{*}, y_{1}^{*}, y_{2}^{*}\right)$ of system (5) is asymptotically stable for $\tau_{2} \in\left[0, \tau_{20}\right)$;

(ii) system (5) undergoes a Hopf bifurcation at the positive equilibrium $E^{*}\left(x_{1}^{*}, x_{2}^{*}, y_{1}^{*}, y_{2}^{*}\right)$ when $\tau_{2}=\tau_{20}$, and a family of periodic solutions bifurcate from $E^{*}\left(x_{1}^{*}, x_{2}^{*}, y_{1}^{*}, y_{2}^{*}\right)$ near $\tau_{2}=\tau_{20}$.

Case $4\left(\tau_{1}=\tau_{2}=\tau>0\right)$. For $\tau_{1}=\tau_{2}=\tau>0$, (11) can be transformed into the following form:

$$
\begin{gathered}
\lambda^{4}+A_{43} \lambda^{3}+A_{42} \lambda^{2}+A_{41} \lambda+A_{40} \\
+\left(B_{43} \lambda^{3}+B_{42} \lambda^{2}+B_{41} \lambda+B_{40}\right) e^{-\lambda \tau} \\
+\left(C_{41} \lambda+C_{40}\right) e^{-2 \lambda \tau}=0
\end{gathered}
$$

where

$$
\begin{gathered}
A_{43}=m_{3}, \quad A_{42}=m_{2}, \quad A_{41}=m_{1}, \quad A_{40}=m_{0}, \\
B_{43}=n_{3}, \quad B_{42}=n_{2}+p_{2}, \\
B_{41}=n_{1}+p_{1}, \quad B_{40}=n_{0}+p_{0}, \\
C_{41}=q_{1}, \quad C_{40}=q_{0} .
\end{gathered}
$$

Multiplying by $e^{\lambda \tau}$, (33) becomes

$$
\begin{aligned}
\left(\lambda^{4}+A_{43} \lambda^{3}+A_{42} \lambda^{2}+A_{41} \lambda+A_{40}\right) e^{\lambda \tau} \\
+\left(C_{41} \lambda+C_{40}\right) e^{-\lambda \tau}+B_{43} \lambda^{3}+B_{42} \lambda^{2} \\
+B_{41} \lambda+B_{40}=0 .
\end{aligned}
$$

Let $\lambda=i \omega(\omega>0)$ be the root of (35). Then, we can get

$$
\begin{aligned}
& \left(\omega^{4}-A_{42} \omega^{2}+A_{40}+C_{40}\right) \cos \tau \omega \\
& +\left(A_{43} \omega^{3}-A_{41} \omega+C_{41} \omega\right) \sin \tau \omega=B_{42} \omega^{2}-B_{40} \\
& \left(\omega^{4}-A_{42} \omega^{2}+A_{40}-C_{40}\right) \sin \tau \omega \\
& \quad-\left(A_{43} \omega^{3}-A_{41} \omega-C_{41} \omega\right) \cos \tau \omega \\
& =B_{43} \omega^{3}-B_{41} \omega .
\end{aligned}
$$


Then, we obtain

$$
\begin{aligned}
& \sin \tau \omega=\frac{A_{7} \omega^{7}+A_{5} \omega^{5}+A_{3} \omega^{3}+A_{1} \omega}{\omega^{8}+B_{6} \omega^{6}+B_{4} \omega^{4}+B_{2} \omega^{2}+B_{0}}, \\
& \cos \tau \omega=\frac{A_{6} \omega^{6}+A_{4} \omega^{4}+A_{2} \omega^{2}+A_{0}}{\omega^{8}+B_{6} \omega^{6}+B_{4} \omega^{4}+B_{2} \omega^{2}+B_{0}},
\end{aligned}
$$

where

$$
\begin{gathered}
A_{0}=\left(C_{40}-A_{40}\right) B_{40}, \\
A_{1}=\left(A_{41}+C_{41}\right) B_{40}-\left(A_{40}+C_{40}\right) B_{41}, \\
A_{2}=A_{40} B_{42}+A_{42} B_{40}+B_{41} C_{41}-A_{41} B_{41}-B_{42} C_{40}, \\
A_{3}=A_{40} B_{43}+A_{42} B_{41}+B_{43} C_{40} \\
-A_{41} B_{42}-A_{43} B_{40}-B_{42} C_{41}, \\
A_{4}=A_{41} B_{43}+A_{43} B_{41}-A_{42} B_{42}-B_{43} C_{41}-B_{40}, \\
A_{5}=A_{43} B_{42}-A_{42} B_{43}-B_{41}, \\
A_{6}=B_{42}-A_{43} B_{43}, \quad A_{7}=B_{43}, \\
B_{0}=A_{40}^{2}-C_{40}^{2}, \quad B_{2}=A_{41}^{2}-C_{41}^{2}-2 A_{40} A_{42}, \\
B_{4}=A_{42}^{2}+2 A_{40}-2 A_{41} A_{43}, \quad B_{6}=A_{43}^{2}-2 A_{42} .
\end{gathered}
$$

It is well known that $\sin ^{2} \tau \omega+\cos ^{2} \tau \omega=1$. Therefore, we have

$$
\begin{gathered}
\omega^{16}+e_{47} \omega^{14}+e_{46} \omega^{12}+e_{45} \omega^{10}+e_{44} \omega^{8} \\
+e_{43} \omega^{6}+e_{42} \omega^{4}+e_{41} \omega^{2}+e_{40}=0
\end{gathered}
$$

where

$$
\begin{gathered}
e_{47}=2 B_{6}-A_{7}^{2}, \quad e_{46}=B_{6}^{2}-A_{6}^{2}+2 B_{4}-2 A_{5} A_{7}, \\
e_{45}=2 B_{2}+2 B_{4} B_{6}-A_{5}^{2}-2 A_{3} A_{7}-2 A_{4} A_{6}, \\
e_{44}=B_{4}^{2}+2 B_{0}+2 B_{2} B_{6}-A_{4}^{2}-2 A_{1} A_{7}-2 A_{3} A_{5}-2 A_{2} A_{6}, \\
e_{43}=2 B_{0} B_{6}+2 B_{2} B_{4}-2 A_{1} A_{5}-2 A_{0} A_{6}-2 A_{2} A_{4}-A_{3}^{2}, \\
e_{42}=B_{2}^{2}-A_{2}^{2}+2 B_{0} B_{4}-2 A_{1} A_{3}-2 A_{0} A_{4}, \\
e_{42}=2 B_{0} B_{2}-2 A_{0} A_{2}-A_{1}^{2}, \quad e_{40}=B_{0}^{2}-A_{0}^{2} .
\end{gathered}
$$

Let $\omega^{2}=v$, then (39) becomes

$$
\begin{gathered}
v^{8}+e_{47} v^{7}+e_{46} v^{6}+e_{45} v^{5}+e_{44} v^{4}+e_{43} v^{3} \\
+e_{42} v^{2}+e_{41} v+e_{40}=0 .
\end{gathered}
$$

If all the parameters of system (5) are given, it is easy to calculate the roots of (41). Thus, we suppose the following:

$\left(H_{41}\right)$ Equation (41) has at least one positive real root.

Without loss of generality, we assume that (41) has eight positive real roots, which are denoted as $v_{1}, v_{2}, v_{3}, \ldots, v_{8}$. Then (39) has eight positive roots $\omega_{k}=\sqrt{v_{k}}, k=1,2,3, \ldots, 8$. And for every fixed $\omega_{k}, k=1,2,3, \ldots, 8$, the corresponding critical value of time delay $\tau_{k}^{(j)}$ is

$$
\begin{array}{r}
\tau_{k}^{(j)}=\frac{1}{\omega_{k}} \arccos \frac{A_{6} \omega_{k}^{6}+A_{4} \omega_{k}^{4}+A_{2} \omega_{k}^{2}+A_{0}}{\omega_{k}^{8}+B_{6} \omega_{k}^{6}+B_{4} \omega_{k}^{4}+B_{2} \omega_{k}^{2}+B_{0}}+\frac{2 j \pi}{\omega_{k}}, \\
k=1,2,3, \ldots, 8 ; j=0,1,2, \ldots
\end{array}
$$

Let

$$
\tau_{0}=\min \left\{\tau_{k}^{(0)}\right\}, \quad \omega_{0}=\left.\omega_{k}\right|_{\tau=\tau_{0}}, \quad k=1,2,3, \ldots, 8 .
$$

Next, we verify the transversality condition. Differentiating both sides of (35) with respect to $\tau$, we get

$$
\begin{aligned}
{\left[\frac{d \lambda}{d \tau}\right]^{-1}=-(} & \left(\left(4 \lambda^{3}+3 A_{43} \lambda^{2}+2 A_{42} \lambda+A_{41}\right) e^{\lambda \tau}\right. \\
& \left.+C_{41} e^{-\lambda \tau}+3 B_{43} \lambda^{2}+2 B_{42} \lambda+B_{41}\right) \\
& \times\left(\lambda \left(\left(\lambda^{4}+A_{43} \lambda^{3}+A_{42} \lambda^{2}+A_{41} \lambda+A_{40}\right)\right.\right. \\
& \left.\left.\left.\times e^{\lambda \tau}-\left(C_{41} \lambda+C_{40}\right) e^{-\lambda \tau}\right)\right)^{-1}\right)-\frac{\tau}{\lambda}
\end{aligned}
$$

Thus,

$$
\operatorname{Re}\left[\frac{d \lambda}{d \tau}\right]_{\tau=\tau_{0}}^{-1}=-\frac{P_{41} Q_{41}+P_{42} Q_{42}}{Q_{41}^{2}+Q_{42}^{2}}
$$

where

$$
\begin{aligned}
P_{41}= & \left(A_{41}+C_{41}-3 A_{43} \omega_{0}^{2}\right) \cos \tau_{0} \omega_{0} \\
& -\left(2 A_{42} \omega_{0}-4 \omega_{0}^{3}\right) \sin \tau_{0} \omega_{0}-3 B_{43} \omega_{0}^{2}+B_{41}, \\
P_{42}= & \left(A_{41}-C_{41}-3 A_{43} \omega_{0}^{2}\right) \sin \tau_{0} \omega_{0} \\
& +\left(2 A_{42} \omega_{0}-4 \omega_{0}^{3}\right) \cos \tau_{0} \omega_{0}+2 B_{42} \omega_{0}, \\
Q_{41}= & \left(A_{43} \omega_{0}^{4}-A_{41} \omega_{0}^{2}-C_{41} \omega_{0}^{2}\right) \cos \tau_{0} \omega_{0} \\
& -\left(\omega_{0}^{5}-A_{42} \omega_{0}^{3}+A_{40} \omega_{0}+C_{40} \omega_{0}\right) \sin \tau_{0} \omega_{0}, \\
Q_{42}= & \left(A_{43} \omega_{0}^{4}-A_{41} \omega_{0}^{2}+C_{41} \omega_{0}^{2}\right) \sin \tau_{0} \omega_{0} \\
& +\left(\omega_{0}^{5}-A_{42} \omega_{0}^{3}+A_{40} \omega_{0}-C_{40} \omega_{0}\right) \cos \tau_{0} \omega_{0} .
\end{aligned}
$$

Obviously, if the condition $\left(H_{42}\right): P_{41} Q_{41}+P_{42} Q_{42} \neq 0$ holds, then $\operatorname{Re}[d \lambda / d \tau]_{\tau=\tau_{0}}^{-1} \neq 0$. Namely, if the condition $\left(H_{42}\right): P_{41} Q_{41}+P_{42} Q_{42} \neq 0$ holds, the transversality condition is satisfied. In conclusion, we have the following results.

Theorem 4. If the conditions $\left(H_{41}\right)-\left(H_{42}\right)$ hold, then

(i) the positive equilibrium $E^{*}\left(x_{1}^{*}, x_{2}^{*}, y_{1}^{*}, y_{2}^{*}\right)$ of system (5) is asymptotically stable for $\tau \in\left[0, \tau_{0}\right)$; 
(ii) system (5) undergoes a Hopf bifurcation at the equilibrium $E^{*}\left(x_{1}^{*}, x_{2}^{*}, y_{1}^{*}, y_{2}^{*}\right)$ when $\tau=\tau_{0}$, and a family of periodic solutions bifurcate from $E^{*}\left(x_{1}^{*}, x_{2}^{*}, y_{1}^{*}, y_{2}^{*}\right)$ near $\tau=\tau_{0}$.

Case $5\left(\tau_{1}>0, \tau_{2}>0\right.$ and $\left.\tau_{1} \in\left(0, \tau_{10}\right)\right)$. We consider system (5) under Case 2. That is, $\tau_{1}$ is in its stable interval and $\tau_{2}$ is considered as a parameter. Let $\lambda=i \omega_{2}^{\prime}\left(\omega_{2}^{\prime}>0\right)$ be the root of (11). Then, we can get

$$
\begin{aligned}
& \Delta_{51} \sin \tau_{2} \omega_{2}^{\prime}+\Delta_{52} \cos \tau_{2} \omega_{2}^{\prime}=\Delta_{53}, \\
& \Delta_{51} \cos \tau_{2} \omega_{2}^{\prime}-\Delta_{52} \sin \tau_{2} \omega_{2}^{\prime}=\Delta_{54},
\end{aligned}
$$

where

$$
\begin{aligned}
\Delta_{51}=q_{1} \omega_{2}^{\prime} \cos \tau_{1} \omega_{2}^{\prime}-q_{0} \sin \tau_{1} \omega_{2}^{\prime}+p_{1} \omega_{2}^{\prime}, \\
\Delta_{52}=q_{1} \omega_{2}^{\prime} \sin \tau_{1} \omega_{2}^{\prime}+q_{0} \cos \tau_{1} \omega_{2}^{\prime}-p_{2}\left(\omega_{2}^{\prime}\right)^{2}+p_{0}, \\
\Delta_{53}=\left(n_{2}\left(\omega_{2}^{\prime}\right)^{2}-n_{0}\right) \cos \tau_{1} \omega_{2}^{\prime}+\left(n_{3}\left(\omega_{2}^{\prime}\right)^{3}-n_{1} \omega_{2}^{\prime}\right) \\
\quad \times \sin \tau_{1} \omega_{2}^{\prime}-\left(\omega_{2}^{\prime}\right)^{4}+m_{2}\left(\omega_{2}^{\prime}\right)^{2}-m_{0}, \\
\Delta_{54}=\left(n_{0}-n_{2}\left(\omega_{2}^{\prime}\right)^{2}\right) \sin \tau_{1} \omega_{2}^{\prime}+\left(n_{3}\left(\omega_{2}^{\prime}\right)^{3}-n_{1} \omega_{2}^{\prime}\right) \\
\quad \times \cos \tau_{1} \omega_{2}^{\prime}+m_{3}\left(\omega_{2}^{\prime}\right)^{3}-m_{1} \omega_{2}^{\prime} .
\end{aligned}
$$

It follows that

$$
c_{0}\left(\omega_{2}^{\prime}\right)+c_{1}\left(\omega_{2}^{\prime}\right) \cos \tau_{1} \omega_{2}^{\prime}+c_{2}\left(\omega_{2}^{\prime}\right) \sin \tau_{1} \omega_{2}^{\prime}=0
$$

with

$$
\begin{aligned}
& c_{0}\left(\omega_{2}^{\prime}\right)=\left(\omega_{2}^{\prime}\right)^{8}+\left(m_{3}^{2}+n_{3}^{2}-2 m_{2}\right)\left(\omega_{2}^{\prime}\right)^{6} \\
&+\left(m_{2}^{2}+n_{2}^{2}-p_{2}^{2}+2 m_{0}-2 m_{1} m_{3}-2 n_{1} n_{3}\right)\left(\omega_{2}^{\prime}\right)^{4} \\
&+\left(m_{1}^{2}+n_{1}^{2}-p_{1}^{2}-q_{1}^{2}-2 m_{0} m_{2}\right. \\
&\left.-2 n_{0} n_{2}+2 p_{0} p_{2}\right)\left(\omega_{2}^{\prime}\right)^{2} \\
&+m_{0}^{2}+n_{0}^{2}-p_{0}^{2}-q_{0}^{2}, \\
& c_{1}\left(\omega_{2}^{\prime}\right)=2\left(m_{3} n_{3}-n_{2}\right)\left(\omega_{2}^{\prime}\right)^{6} \\
&+2\left(m_{2} n_{2}+n_{0}-m_{3} n_{1}-m_{1} n_{3}\right)\left(\omega_{2}^{\prime}\right)^{4} \\
&+2\left(m_{1} n_{1}-m_{0} n_{2}-m_{2} n_{0}-p_{1} q_{1}+p_{2} q_{0}\right)\left(\omega_{2}^{\prime}\right)^{2} \\
&+2\left(m_{0} n_{0}-p_{0} q_{0}\right), \\
&+2\left(m_{2} n_{3}-m_{3} n_{2}+n_{1}\right)\left(\omega_{2}^{\prime}\right)^{5} \\
& c_{2}\left(\omega_{2}^{\prime}\right)=-2 n_{3}\left(\omega_{2}^{\prime}\right)^{7} \\
&
\end{aligned}
$$

$$
\begin{aligned}
& +2\left(m_{3} n_{0}+m_{1} n_{2}-m_{0} n_{3}\right. \\
& \left.\quad-m_{2} n_{1}+p_{2} q_{1}\right)\left(\omega_{2}^{\prime}\right)^{3} \\
& +2\left(m_{0} n_{1}-m_{1} n_{0}+p_{1} q_{0}-p_{0} q_{1}\right) \omega_{2}^{\prime} .
\end{aligned}
$$

Suppose that $\left(H_{51}\right)$ : Equation (49) has at least finite positive real roots. If the condition $\left(H_{51}\right)$ holds, we denote the positive roots of $(49)$ as $\omega_{21}^{\prime}, \omega_{22}^{\prime}, \ldots, \omega_{2 k}^{\prime}$. Then, for every fixed $\omega_{2 i}^{\prime}(i=1,2,3, \ldots, k)$, the corresponding critical value of time delay $\left\{\tau_{2 i}^{\prime(j)} \mid j=0,1,2, \ldots\right\}$ is

$$
\tau_{2 i}^{\prime(j)}=\frac{1}{\omega_{2 i}^{\prime}} \arccos \frac{\Delta_{51} \Delta_{54}+\Delta_{52} \Delta_{53}}{\Delta_{51}^{2}+\Delta_{52}^{2}}+\frac{2 j \pi}{\omega_{2 i}^{\prime}},
$$

Let

$$
\tau_{2}^{*}=\min \left\{\tau_{2 i}^{\prime^{(0)}} \mid i=1,2,3, \ldots, k,\right\}, \quad \omega_{2}^{*}=\left.\omega_{2 i}^{\prime}\right|_{\tau_{2}=\tau_{2}^{*}} .
$$

Differentiating (11) regarding $\tau_{2}$, we obtain

$$
\begin{aligned}
& {\left[\frac{d \lambda}{d \tau_{2}}\right]^{-1}} \\
& =\frac{g_{0}(\lambda)+g_{1}(\lambda) e^{-\lambda \tau_{1}}+g_{2}(\lambda) e^{-\lambda \tau_{2}}+g_{3}(\lambda) e^{-\lambda\left(\tau_{1}+\tau_{2}\right)}}{h_{2}(\lambda) e^{-\lambda \tau_{2}}+h_{3}(\lambda) e^{-\lambda\left(\tau_{1}+\tau_{2}\right)}} \\
& \quad-\frac{\tau_{2}}{\lambda}
\end{aligned}
$$

with

$$
\begin{gathered}
g_{0}(\lambda)=4 \lambda^{3}+3 m_{3} \lambda^{2}+2 m_{2} \lambda+m_{1}, \\
g_{1}(\lambda)=-\tau_{1} n_{3} \lambda^{3}+\left(3 n_{3}-\tau_{1} n_{2}\right) \lambda^{2} \\
+\left(2 n_{2}-\tau_{1} n_{1}\right) \lambda+n_{1}-\tau_{1} n_{0}, \\
g_{2}(\lambda)=2 p_{2} \lambda+p_{1}, \quad g_{3}(\lambda)=q_{1}, \\
h_{2}(\lambda)=p_{2} \lambda^{3}+p_{1} \lambda^{2}+p_{0} \lambda, \quad
\end{gathered}
$$

Hence,

$$
\operatorname{Re}\left[\frac{d \lambda}{d \tau_{2}}\right]_{\tau_{2}=\tau_{2}^{*}}^{-1}=-\frac{P_{51} Q_{51}-P_{52} Q_{52}}{Q_{51}^{2}+Q_{52}^{2}},
$$

where

$$
\begin{aligned}
P_{51}= & \left(2 p_{2} \omega_{2}^{*}-q_{1} \sin \tau_{1} \omega_{2}^{*}\right) \sin \tau_{2}^{*} \omega_{2}^{*} \\
& +\left(p_{1}+q_{1} \cos \tau_{1} \omega_{2}^{*}\right) \cos \tau_{2}^{*} \omega_{2}^{*} \\
& +\left(\tau_{1} n_{3}\left(\omega_{2}^{*}\right)^{3}+\left(2 n_{2}-\tau_{1} n_{1}\right) \omega_{2}^{*}\right) \sin \tau_{1} \omega_{2}^{*} \\
& +\left(\left(\tau_{1} n_{2}-3 n_{3}\right)\left(\omega_{2}^{*}\right)^{2}+n_{1}-\tau_{1} n_{0}\right) \cos \tau_{1} \omega_{2}^{*} \\
& -3 m_{3}\left(\omega_{2}^{*}\right)^{2}+m_{1},
\end{aligned}
$$




$$
\begin{aligned}
P_{52}= & \left(2 p_{2} \omega_{2}^{*}-q_{1} \sin \tau_{1} \omega_{2}^{*}\right) \cos \tau_{2}^{*} \omega_{2}^{*} \\
& -\left(p_{1}+q_{1} \cos \tau_{1} \omega_{2}^{*}\right) \sin \tau_{2}^{*} \omega_{2}^{*} \\
& +\left(\tau_{1} n_{3}\left(\omega_{2}^{*}\right)^{3}+\left(2 n_{2}-\tau_{1} n_{1}\right) \omega_{2}^{*}\right) \cos \tau_{1} \omega_{2}^{*} \\
& -\left(\left(\tau_{1} n_{2}-3 n_{3}\right)\left(\omega_{2}^{*}\right)^{2}+n_{1}-\tau_{1} n_{0}\right) \sin \tau_{1} \omega_{2}^{*} \\
& -4\left(\omega_{2}^{*}\right)^{3}+2 m_{2} \omega_{2}^{*}, \\
Q_{51}= & \left(p_{2}\left(\omega_{2}^{*}\right)^{3}-p_{0} \omega_{2}^{*}-q_{1}\left(\omega_{2}^{*}\right)^{2} \sin \tau_{1} \omega_{2}^{*}\right. \\
& \left.-q_{0} \omega_{2}^{*} \cos \tau_{1} \omega_{2}^{*}\right) \sin \tau_{2}^{*} \omega_{2}^{*} \\
& +\left(p_{1}\left(\omega_{2}^{*}\right)^{2}+q_{1}\left(\omega_{2}^{*}\right)^{2} \cos \tau_{1} \omega_{2}^{*}\right. \\
& \left.\quad-q_{0} \omega_{2}^{*} \sin \tau_{1} \omega_{2}^{*}\right) \cos \tau_{2}^{*} \omega_{2}^{*}, \\
Q_{52}= & \left(p_{2}\left(\omega_{2}^{*}\right)^{3}-p_{0} \omega_{2}^{*}-q_{1}\left(\omega_{2}^{*}\right)^{2} \sin \tau_{1} \omega_{2}^{*}\right. \\
& \left.-q_{0} \omega_{2}^{*} \cos \tau_{1} \omega_{2}^{*}\right) \cos \tau_{2}^{*} \omega_{2}^{*} \\
& -\left(p_{1}\left(\omega_{2}^{*}\right)^{2}+q_{1}\left(\omega_{2}^{*}\right)^{2} \cos \tau_{1} \omega_{2}^{*}\right. \\
& \left.-q_{0} \omega_{2}^{*} \sin \tau_{1} \omega_{2}^{*}\right) \sin \tau_{2}^{*} \omega_{2}^{*} .
\end{aligned}
$$

Clearly, if the condition $\left(H_{52}\right) P_{51} Q_{51}-P_{52} Q_{52} \neq 0$ holds, then $\operatorname{Re}\left[d \lambda / d \tau_{2}\right]_{\tau_{2}=\tau_{2}^{*}}^{-1} \neq 0$. Thus, we have the following results.

Theorem 5. If the conditions $\left(H_{51}\right)-\left(H_{52}\right)$ hold and $\tau_{1} \in$ $\left(0, \tau_{10}\right)$, then

(i) the positive equilibrium $E^{*}\left(x_{1}^{*}, x_{2}^{*}, y_{1}^{*}, y_{2}^{*}\right)$ of system (5) is asymptotically stable for $\tau_{2} \in\left[0, \tau_{2}^{*}\right)$;

(ii) system (5) undergoes a Hopf bifurcation at the equilibrium $E^{*}\left(x_{1}^{*}, x_{2}^{*}, y_{1}^{*}, y_{2}^{*}\right)$ when $\tau_{2}=\tau_{2}^{*}$, and a family of periodic solutions bifurcate from $E^{*}\left(x_{1}^{*}, x_{2}^{*}, y_{1}^{*}, y_{2}^{*}\right)$ near $\tau_{2}=\tau_{2}^{*}$.

\section{Stability of the Bifurcating Periodic Solutions}

In this section, we will determine the direction of Hopf bifurcation and stability of the bifurcated periodic solutions of system (5) with respect to $\tau_{2}$ for $\tau_{1} \in\left(0, \tau_{10}\right)$ by using the normal form method and center manifold theorem introduced by Hassard et al. in [25]. Without loss of generality, we assume that $\tau_{1}^{*}<\tau_{2}^{*}$, where $\tau_{1}^{*} \in\left(0, \tau_{10}\right)$.

Let $\tau_{2}=\tau_{2}^{*}+\mu, \mu \in R$. Then, $\mu$ is the Hopf bifurcation value of system (5). Rescaling the time $t \rightarrow t / \tau_{2}$, then system (5) can be written as

$$
\dot{u}(t)=L_{\mu} u_{t}+F\left(\mu, u_{t}\right)
$$

where

$$
\begin{aligned}
L_{\mu} \phi=\left(\tau_{2}^{*}+\mu\right)\left(A^{\prime} \phi(0)+B^{\prime} \phi\left(-\frac{\tau_{1}^{*}}{\tau_{2}^{*}}\right)+C^{\prime} \phi(-1)\right), \\
\begin{aligned}
F(\mu, \phi)= & \left(\tau_{2}^{*}+\mu\right) \\
& \times\left(\begin{array}{c}
g_{1} x_{1}(t) y_{2}(t)+g_{2} x_{1}(t) x_{1}\left(t-\tau_{1}\right) \\
0 \\
h_{1} x_{1}\left(t-\tau_{2}\right) y_{2}\left(t-\tau_{2}\right) \\
0
\end{array}\right)
\end{aligned}
\end{aligned}
$$

with

$$
\begin{gathered}
\phi(\theta)=\left(\phi_{1}(\theta), \phi_{2}(\theta), \phi_{3}(\theta), \phi_{4}(\theta)\right)^{T} \in C\left([-1,0], R^{4}\right), \\
A^{\prime}=\left(\begin{array}{cccc}
a_{11} & a_{12} & 0 & a_{14} \\
a_{21} & a_{22} & 0 & 0 \\
0 & 0 & a_{33} & 0 \\
0 & 0 & a_{43} & a_{44}
\end{array}\right) \\
B^{\prime}=\left(\begin{array}{cccc}
b_{11} & 0 & 0 & 0 \\
0 & 0 & 0 & 0 \\
0 & 0 & 0 & 0 \\
0 & 0 & 0 & 0
\end{array}\right) \\
C^{\prime}=\left(\begin{array}{cccc}
0 & 0 & 0 & 0 \\
0 & 0 & 0 & 0 \\
c_{31} & 0 & 0 & c_{34} \\
0 & 0 & 0 & 0
\end{array}\right)
\end{gathered}
$$

Using the Riesz representation theorem, there exists a $4 \times 4$ matrix function $\eta(\theta, \mu):[-1,0] \rightarrow R^{4}$ whose elements are of bounded variation, such that

$$
L_{\mu} \phi=\int_{-1}^{0} d \eta(\theta, \mu) \phi(\theta), \quad \phi \in C\left([-1,0], R_{4}\right) .
$$

In fact, we can choose

$$
\eta(\theta, \mu)= \begin{cases}\left(\tau_{2}^{*}+\mu\right)\left(A^{\prime}+B^{\prime}+C^{\prime}\right), & \theta=0, \\ \left(\tau_{2}^{*}+\mu\right)\left(B^{\prime}+C^{\prime}\right), & \theta \in\left[-\frac{\tau_{1}^{*}}{\tau_{2}^{*}}, 0\right), \\ \left(\tau_{2}^{*}+\mu\right) C^{\prime}, & \theta \in\left(-1,-\frac{\tau_{1}^{*}}{\tau_{2}^{*}}\right), \\ 0, & \theta=-1 .\end{cases}
$$

For $\phi \in C\left([-1,0], R^{4}\right)$, we define

$$
\begin{gathered}
A(\mu) \phi= \begin{cases}\frac{d \phi(\theta)}{d \theta}, & -1 \leq \theta<0, \\
\int_{-1}^{0} d \eta(\theta, \mu) \phi(\theta), & \theta=0,\end{cases} \\
R(\mu) \phi= \begin{cases}0, & -1 \leq \theta<0, \\
F(\mu, \phi), & \theta=0 .\end{cases}
\end{gathered}
$$



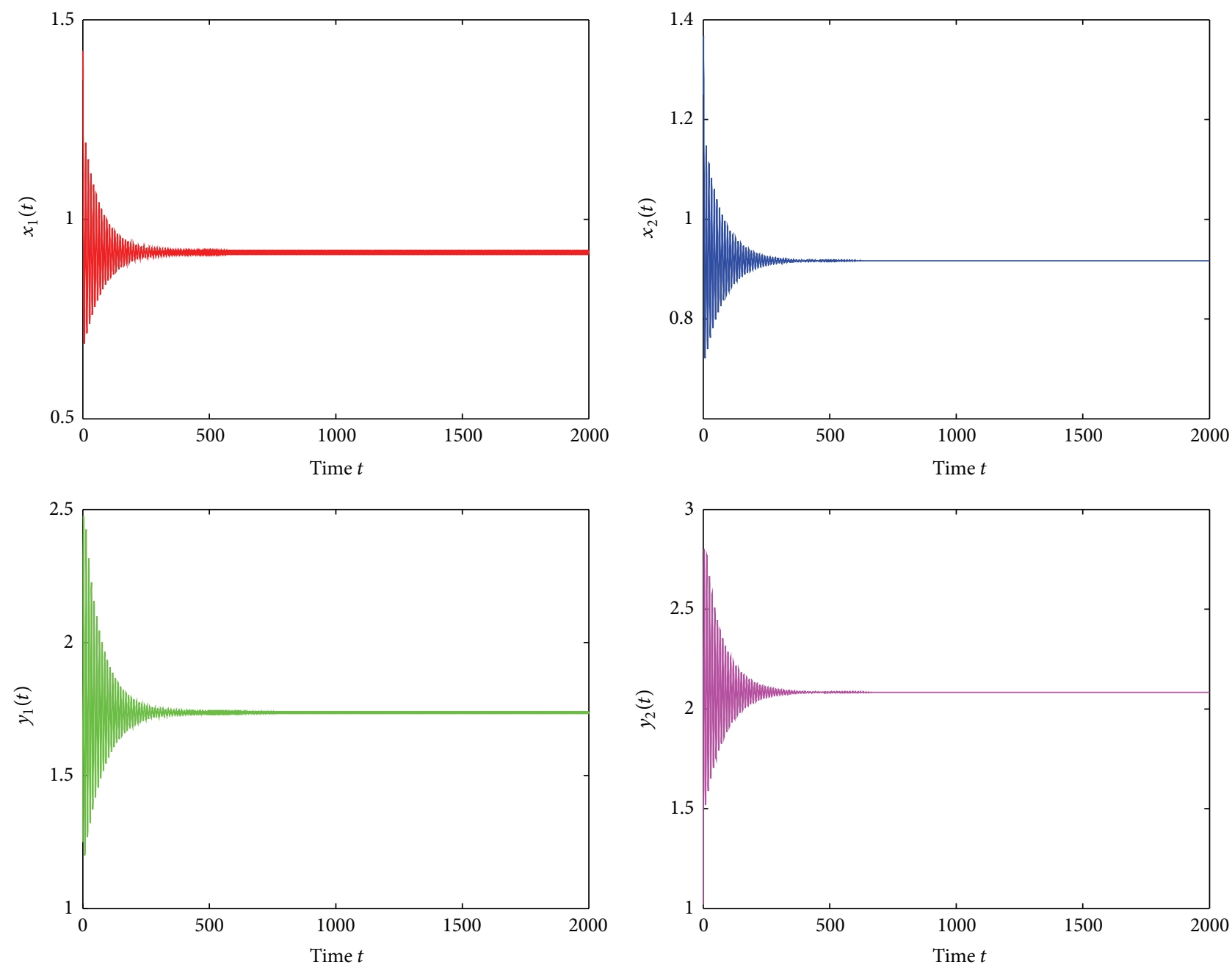

FIgURE 1: The track of the states $x_{1}, x_{2}, y_{1}, y_{2}$ for $\tau_{1}=4.2500<\tau_{10}=4.5061$.

Then system (57) can be transformed into the following operator equation:

$$
\dot{u}(t)=A(\mu) u_{t}+R(\mu) u_{t}
$$

where $u_{t}=u(t+\theta)=\left(u_{1}(t+\theta), u_{2}(t+\theta), u_{3}(t+\theta), u_{4}(t+\theta)\right)$.

The adjoint operator $A^{*}$ of $\mathrm{A}$ is defined by

$$
A^{*}(\varphi)= \begin{cases}-\frac{d \varphi(s)}{d s}, & 0<s \leq 1, \\ \int_{-1}^{0} d \eta^{T}(s, \mu) \varphi(-s), & s=0,\end{cases}
$$

associated with a bilinear inner product:

$$
\begin{aligned}
\langle\varphi(s), \phi(\theta)\rangle= & \bar{\varphi}(0) \phi(0) \\
& -\int_{\theta=-1}^{0} \int_{\xi=0}^{\theta} \bar{\varphi}(\xi-\theta) d \eta(\theta) \phi(\xi) d \xi,
\end{aligned}
$$

where $\eta(\theta)=\eta(\theta, 0)$.

By the discussion above, we know that $\pm i \tau_{2}^{*} \omega_{2}^{*}$ are eigenvalues of $A(0)$ and they are also eigenvalues of $A^{*}(0)$. We

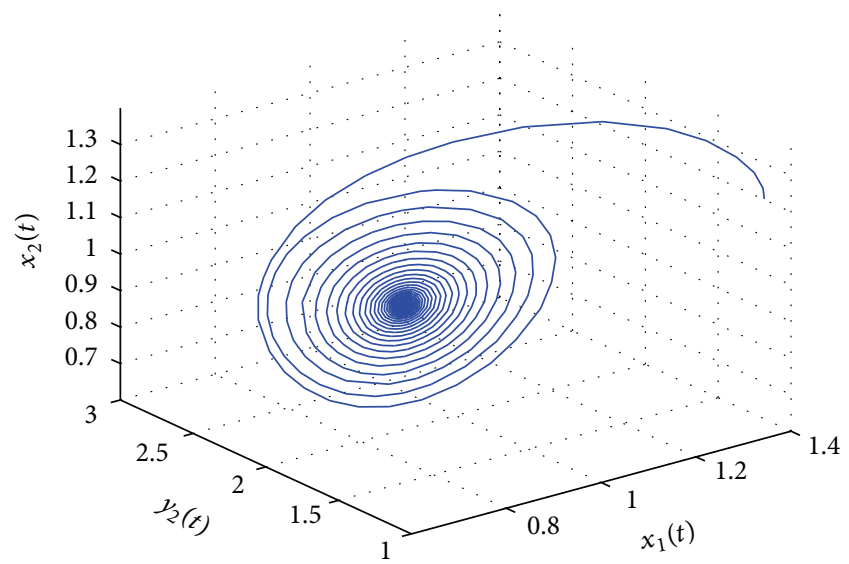

FIGURE 2: The phase plot of the states $x_{1}, x_{2}, y_{2}$ for $\tau_{1}=4.2500<$ $\tau_{10}=4.5061$.

assume that $q(\theta)=\left(1, q_{2}, q_{3}, q_{4}\right)^{T} e^{i \tau_{2}^{*} \omega_{2}^{*} \theta}$ are the eigenvectors of $A(0)$ belonging to the eigenvalue $+i \tau_{2}^{*} \omega_{2}^{*}$ and $q^{*}(s)=$ 
$D\left(1, q_{2}^{*}, q_{3}^{*}, q_{4}^{*}\right) e^{i \tau_{2}^{*} \omega_{2}^{*} s}$ are the eigenvectors of $A^{*}(0)$ belonging to the eigenvalue $-i \tau_{2}^{*} \omega_{2}^{*}$.

By a simple computation, we can get

$$
\begin{gathered}
q_{2}=\frac{a_{21}}{i \omega_{2}^{*}-a_{22}}, \\
q_{3}=\frac{c_{34}\left(i \omega_{2}^{*}-a_{11}\right)+a_{14} c_{31}-b_{11} c_{34} e^{-i \tau_{1}^{*} \omega_{2}^{*}}}{a_{14}\left(i \omega_{2}^{*}-a_{33}\right) e^{i \tau_{2}^{*} \omega_{2}^{*}}} \\
-\frac{a_{12} a_{21} c_{34}}{a_{14}\left(i \omega_{2}^{*}-a_{22}\right)\left(i \omega_{2}^{*}-a_{33}\right) e^{i \tau_{2}^{*} \omega_{2}^{*}}} \\
q_{4}=\left(\left(i \omega_{2}^{*}-a_{11}\right)\left(i \omega_{2}^{*}-a_{22}\right)-a_{12} a_{21}\right. \\
\left.-b_{11}\left(i \omega_{2}^{*}-a_{22}\right) e^{-i \tau_{1}^{*} \omega_{2}^{*}}\right) \times\left(a_{14}\left(i \omega_{2}^{*}-a_{22}\right)\right)^{-1} \\
q_{3}^{*}=-\left(\left(i \omega_{2}^{*}+a_{11}\right)\left(i \omega_{2}^{*}+a_{22}\right)-a_{12} a_{21}+b_{11}\right. \\
\left.\times\left(i \omega_{2}^{*}+a_{22}\right) e^{i \tau_{1}^{*} \omega_{2}^{*}}\right) \times\left(c_{31}\left(i \omega_{2}^{*}+a_{33}\right) e^{i \tau_{2}^{*} \omega_{2}^{*}}\right)^{-1} \\
q_{34}^{*}\left(i \omega_{2}^{*}+a_{11}\right)-a_{14} c_{31}-b_{11} c_{34} e^{i \tau_{1}^{*} \omega_{2}^{*}} \\
c_{31}\left(i \omega_{2}^{*}+a_{44}\right) \\
a_{12} a_{21} c_{34} \\
q_{31}^{*}=\frac{c_{34}\left(i \omega_{2}^{*}+a_{22}\right)\left(i \omega_{2}^{*}+a_{44}\right)}{}
\end{gathered}
$$

From (65), we can get

$$
\begin{aligned}
\left\langle q^{*}(s), q(\theta)\right\rangle= & \bar{q}^{*}(0) q(0) \\
& -\int_{\theta=-1}^{0} \int_{\xi=0}^{\theta} \bar{q}^{*}(\xi-\theta) d \eta(\theta) q(\xi) d \xi \\
= & \bar{D}\left(1, \bar{q}_{2}^{*}, \bar{q}_{3}^{*}, \bar{q}_{4}^{*}\right)\left(1, q_{2}, q_{3}, q_{4}\right)^{T} \\
& -\bar{D} \int_{\theta=-1}^{0} \int_{\xi=0}^{\theta}\left(1, \bar{q}_{2}^{*}, \bar{q}_{3}^{*}, \bar{q}_{4}^{*}\right) \\
= & \times e^{i \tau_{2}^{*} \omega_{2}^{*}(\theta-\xi)} d \eta(\theta) \\
& \times\left(1, q_{2}, q_{3}, q_{4}\right)^{T} e^{i \tau_{2}^{*} \omega_{2}^{*} \xi} d \xi \\
& \quad+q_{-1} \bar{q}_{2}^{*} \bar{q}_{2}^{*}+q_{3} \bar{q}_{3}^{*}+q_{4} \bar{q}_{4}^{*}-\left(1, \bar{q}_{2}^{*}, \bar{q}_{3}^{*}, \bar{q}_{4}^{*}\right) \\
= & \bar{D}\left[1+q_{2} \bar{q}_{2}^{*}+q_{3} \bar{q}_{3}^{*}+q_{4} \bar{q}_{4}^{*}+b_{11} \tau_{1}^{*} e^{-i \tau_{1}^{*} \omega_{2}^{*}}\right. \\
& \left.+\bar{q}_{3}^{*} \tau_{2}^{*} e^{-i \tau_{2}^{*} \omega_{2}^{*}}\left(c_{31}+c_{34} q_{4}\right)\right] .
\end{aligned}
$$

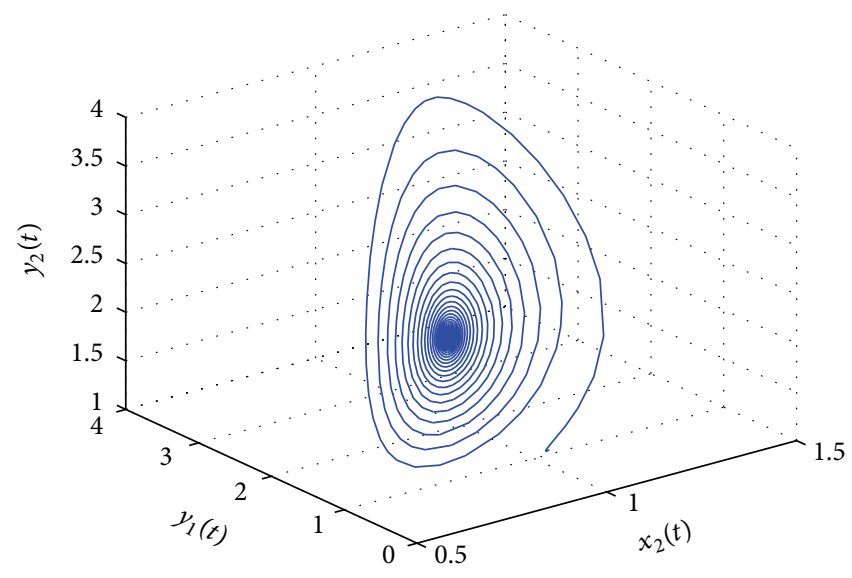

FIgURE 3: The phase plot of the states $x_{2}, y_{1}, y_{2}$ for $\tau_{1}=4.2500<$ $\tau_{10}=4.5061$.

Normalizing $q$ and $q^{*}$ by the condition $\left\langle q^{*}, q\right\rangle=1$ and $\left\langle q^{*}, \bar{q}\right\rangle$, one can get

$$
\begin{gathered}
\bar{D}=\left[1+q_{2} \bar{q}_{2}^{*}+q_{3} \bar{q}_{3}^{*}+q_{4} \bar{q}_{4}^{*}+b_{11} \tau_{1}^{*} e^{-i \tau_{1}^{*} \omega_{2}^{*}}\right. \\
\left.+\bar{q}_{3}^{*} \tau_{2}^{*} e^{-i \tau_{2}^{*} \omega_{2}^{*}}\left(c_{31}+c_{34} q_{4}\right)\right]^{-1} .
\end{gathered}
$$

Next, we can get the coefficients determining the direction of the Hopf bifurcation and the stability of the bifurcated periodic solutions by the algorithms given in [26]:

$$
\begin{aligned}
& g_{20}=2 \tau_{2}^{*} \bar{D}\left[g_{1} q^{(4)}(0)+g_{2} q^{(1)}\left(-\frac{\tau_{1}^{*}}{\tau_{2}^{*}}\right)\right. \\
& \left.+h_{1} \bar{q}_{3}^{*} q^{(1)}(-1) q^{(4)}(-1)\right] \\
& g_{11}=\tau_{2}^{*} \bar{D}\left[g_{1}\left(\bar{q}^{(4)}(0)+q^{(4)}(0)\right)\right. \\
& +g_{2}\left(\bar{q}^{(1)}\left(-\frac{\tau_{1}^{*}}{\tau_{2}^{*}}\right)+q^{(1)}\left(-\frac{\tau_{1}^{*}}{\tau_{2}^{*}}\right)\right) \\
& +h_{1} \bar{q}_{3}^{*}\left(q^{(1)}(-1) \bar{q}^{(4)}(-1)\right. \\
& \left.\left.+\bar{q}^{(1)}(-1) q^{(4)}(-1)\right)\right] \\
& g_{02}=2 \tau_{2}^{*} \bar{D}\left[g_{1} \bar{q}^{(4)}(0)+g_{2} \bar{q}^{(1)}\left(-\frac{\tau_{1}^{*}}{\tau_{2}^{*}}\right)\right. \\
& \left.+h_{1} \bar{q}_{3}^{*} \bar{q}^{(1)}(-1) \bar{q}^{(4)}(-1)\right], \\
& g_{21}=2 \tau_{2}^{*} \bar{D}\left[g _ { 1 } \left(W_{11}^{(1)}(0) q^{(4)}(0)+\frac{1}{2} W_{20}^{(1)}(0) \bar{q}^{(4)}(0)\right.\right. \\
& \left.+W_{11}^{(4)}(0)+\frac{1}{2} W_{20}^{(4)}(0)\right) \\
& +g_{2}\left(W_{11}^{(1)}(0) q^{(1)}\left(-\frac{\tau_{1}^{*}}{\tau_{2}^{*}}\right)+\frac{1}{2} W_{20}^{(1)}(0) \bar{q}^{(1)}\right. \\
& \times\left(-\frac{\tau_{1}^{*}}{\tau_{2}^{*}}\right)+W_{11}^{(1)}\left(-\frac{\tau_{1}^{*}}{\tau_{2}^{*}}\right)
\end{aligned}
$$



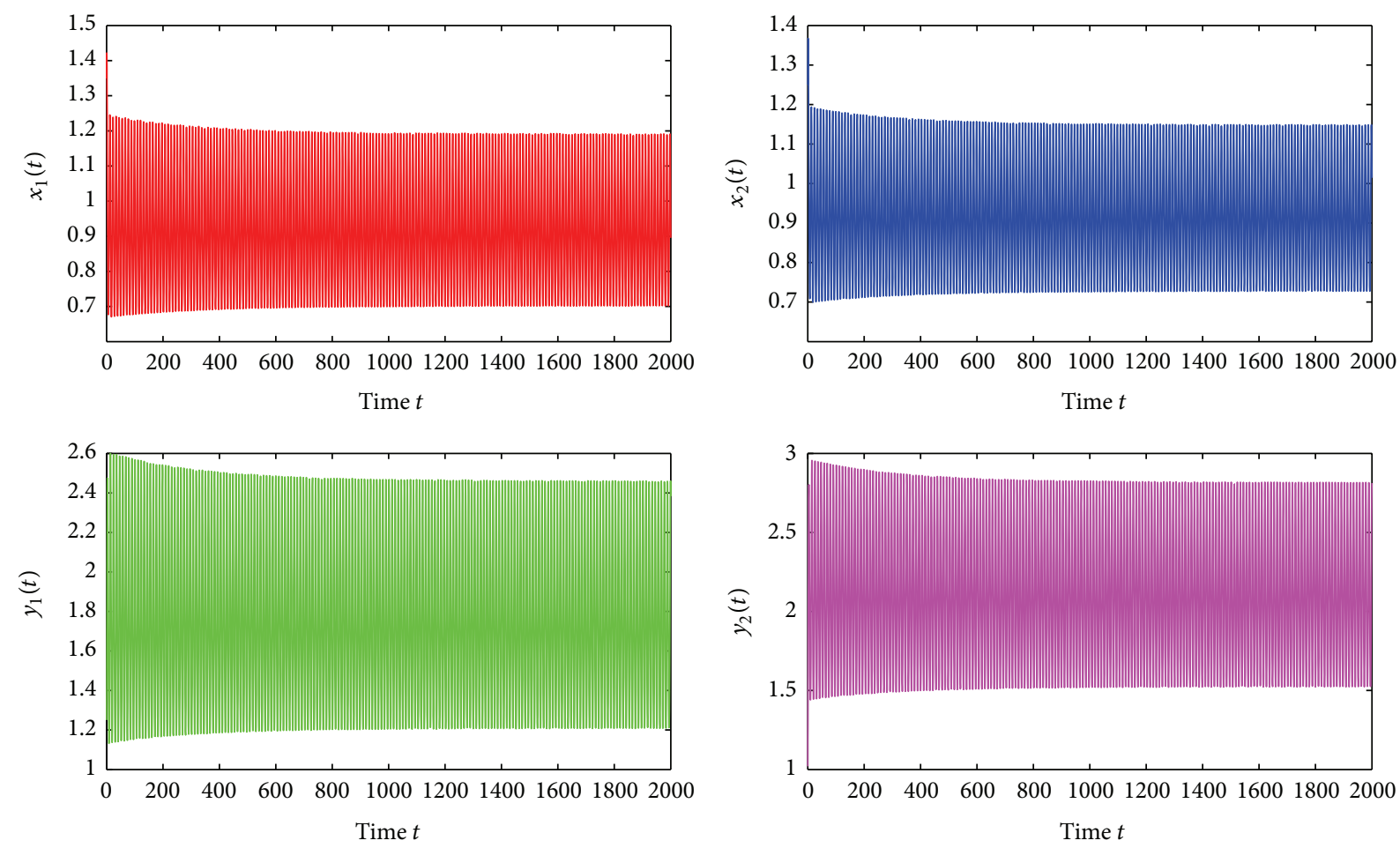

FIgURE 4: The track of the states $x_{1}, x_{2}, y_{1}, y_{2}$ for $\tau_{1}=4.71500>\tau_{10}=4.5061$.

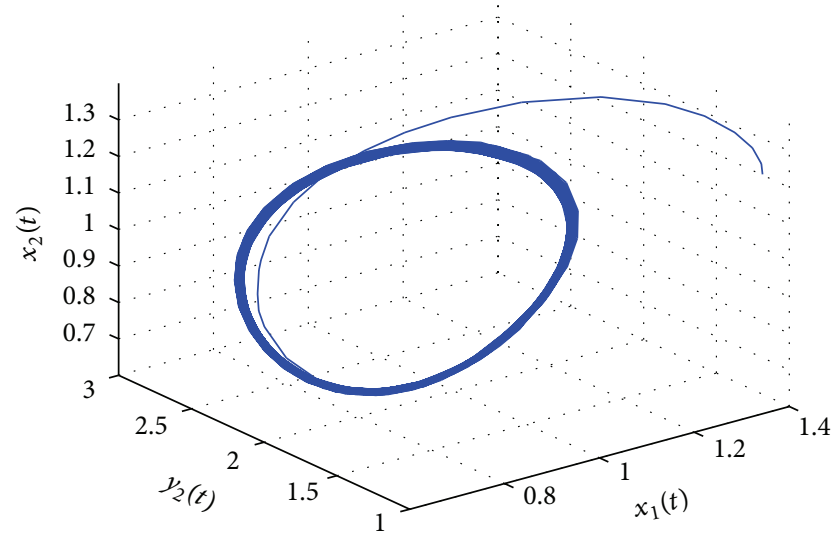

Figure 5: The phase plot of the states $x_{1}, x_{2}, y_{2}$ for $\tau_{1}=4.71500>$ $\tau_{10}=4.5061$.

$$
\begin{aligned}
&+\left.\frac{1}{2} W_{20}^{(1)}\left(-\frac{\tau_{1}^{*}}{\tau_{2}^{*}}\right)\right) \\
&+h_{1} \bar{q}_{3}^{*}\left(W_{11}^{(1)}(-1) q^{(4)}(-1)\right. \\
&+\frac{1}{2} W_{20}^{(1)}(-1) \bar{q}^{(4)}(-1) \\
&+W_{11}^{(4)}(-1) q^{(1)}(-1) \\
&\left.\left.+\frac{1}{2} W_{20}^{(4)}(-1) \bar{q}^{(1)}(-1)\right)\right]
\end{aligned}
$$

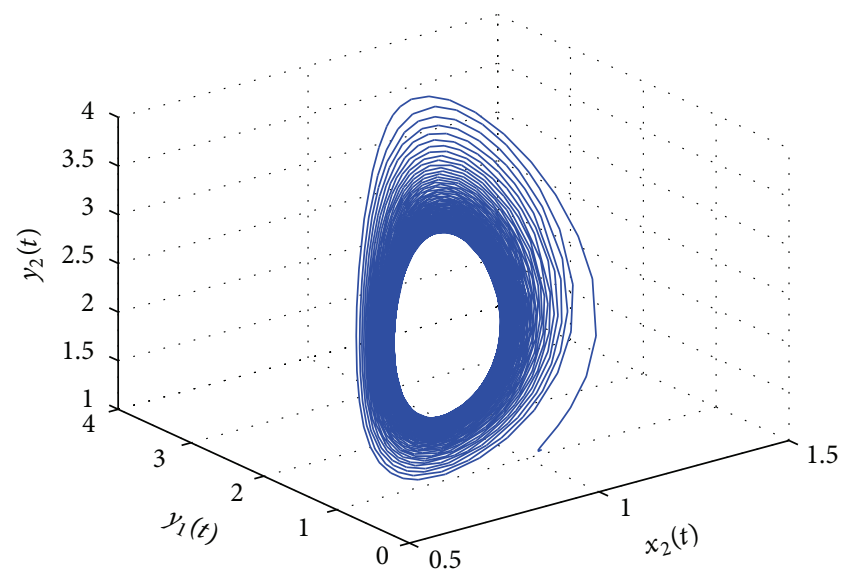

FIGURE 6: The phase plot of the states $x_{2}, y_{1}, y_{2}$ for $\tau_{1}=4.71500>$ $\tau_{10}=4.5061$.

with

$$
\begin{array}{r}
W_{20}(\theta)=\frac{i g_{20} q(0)}{\tau_{2}^{*} \omega_{2}^{*}} e^{i \tau_{2}^{*} \omega_{2}^{*} \theta}+\frac{i \bar{g}_{02} \bar{q}(0)}{3 \tau_{2}^{*} \omega_{2}^{*}} e^{-i \tau_{2}^{*} \omega_{2}^{*} \theta} \\
+E_{1} e^{2 i \tau_{2}^{*} \omega_{2}^{*} \theta}, \\
W_{11}(\theta)=-\frac{i g_{11} q(0)}{\tau_{2}^{*} \omega_{2}^{*}} e^{i \tau_{2}^{*} \omega_{2}^{*} \theta}+\frac{i \bar{g}_{11} \bar{q}(0)}{\tau_{2}^{*} \omega_{2}^{*}} e^{-i \tau_{2}^{*} \omega_{2}^{*} \theta}+E_{2},
\end{array}
$$

where $E_{1}$ and $E_{2}$ can be determined by the following equa tions, respectively: 

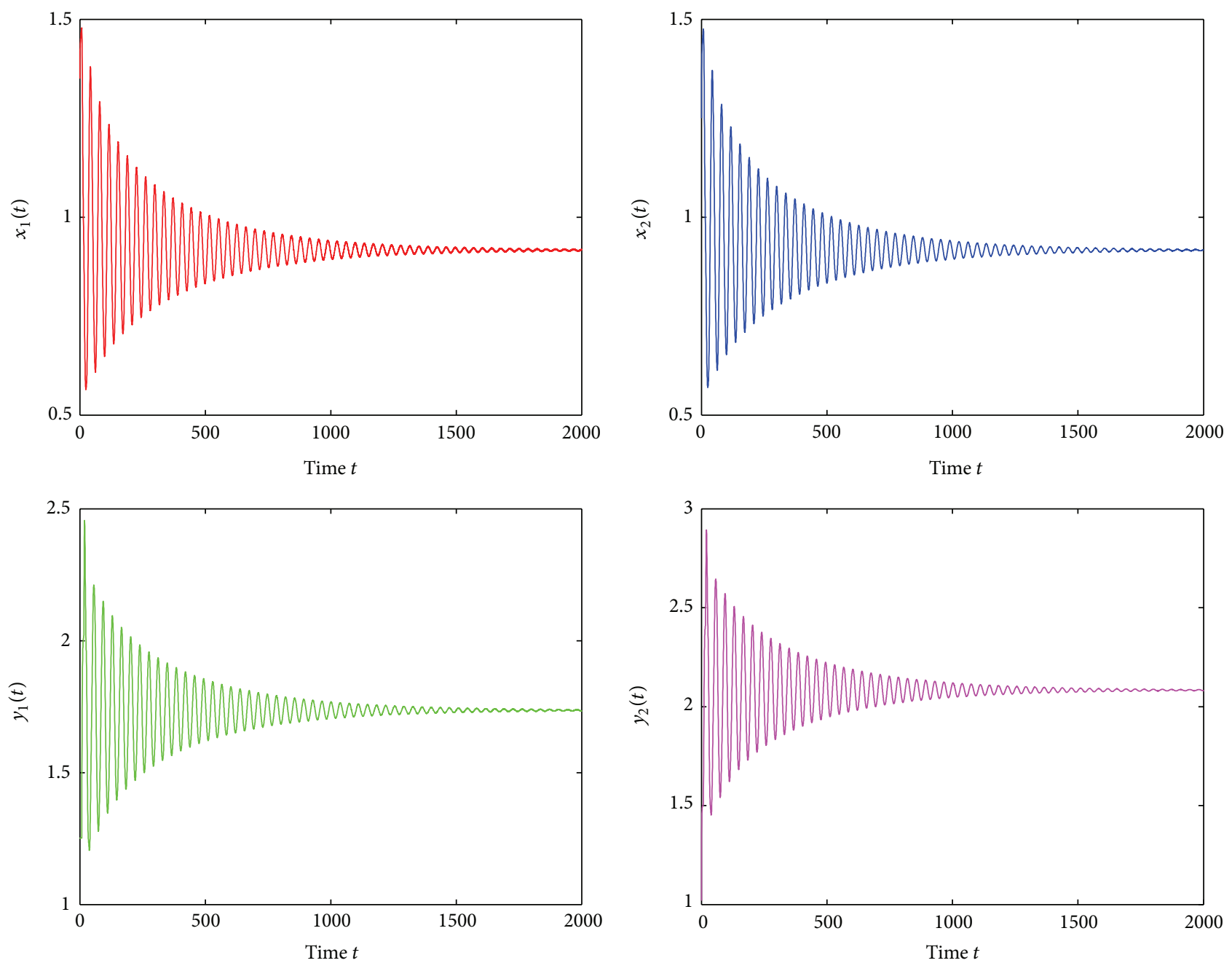

Figure 7: The track of the states $x_{1}, x_{2}, y_{1}, y_{2}$ for $\tau_{2}=7.7500<\tau_{20}=9.9183$.

$$
\begin{aligned}
& E_{1}=2\left(\begin{array}{cccc}
2 i \omega_{2}^{*}-a_{11}-b_{11} e^{-2 i \tau_{1}^{*} \omega_{2}^{*}} & -a_{12} & 0 & -a_{14} \\
-a_{21} & 2 i \omega_{2}^{*}-a_{22} & 0 & 0 \\
-c_{31} e^{-2 i \tau_{2}^{*} \omega_{2}^{*}} & 0 & 2 i \omega_{2}^{*}-a_{33} & -c_{34} e^{-2 i \tau_{2}^{*} \omega_{2}^{*}} \\
0 & 0 & -a_{43} & 2 i \omega_{2}^{*}-a_{44}
\end{array}\right)^{-1} \times\left(\begin{array}{c}
g_{1} q^{(4)}(0)+g_{2} q^{(1)}\left(-\frac{\tau_{1}^{*}}{\tau_{2}^{*}}\right) \\
0 \\
h_{1} q^{(1)}(-1) q^{(4)}(-1) \\
0
\end{array}\right) \text {, } \\
& E_{2}=-\left(\begin{array}{cccc}
a_{11}+b_{11} & a_{12} & 0 & a_{14} \\
a_{21} & a_{22} & 0 & 0 \\
c_{31} & 0 & a_{33} & c_{34} \\
0 & 0 & a_{43} & a_{44}
\end{array}\right)^{-1} \times\left(\begin{array}{c}
g_{1}\left(\bar{q}^{(4)}(0)+q^{(4)}(0)\right)+g_{2}\left(\bar{q}^{(1)}\left(-\frac{\tau_{1}^{*}}{\tau_{2}^{*}}\right)+q^{(1)}\left(-\frac{\tau_{1}^{*}}{\tau_{2}^{*}}\right)\right) \\
0 \\
h_{1}\left(q^{(1)}(-1) \bar{q}^{(4)}(-1)+\bar{q}^{(1)}(-1) q^{(4)}(-1)\right) \\
0
\end{array}\right) .
\end{aligned}
$$

Therefore, we can calculate the following values:

$$
\begin{gathered}
C_{1}(0)=\frac{i}{2 \tau_{2}^{*} \omega_{2}^{*}}\left(g_{11} g_{20}-2\left|g_{11}\right|^{2}-\frac{\left|g_{02}\right|^{2}}{3}\right)+\frac{g_{21}}{2}, \\
\mu_{2}=-\frac{\operatorname{Re}\left\{C_{1}(0)\right\}}{\operatorname{Re}\left\{\lambda^{\prime}\left(\tau_{2}^{*}\right)\right\}},
\end{gathered}
$$

$$
\begin{gathered}
\beta_{2}=2 \operatorname{Re}\left\{C_{1}(0)\right\} \\
T_{2}=-\frac{\operatorname{Im}\left\{C_{1}(0)\right\}+\mu_{2} \operatorname{Im}\left\{\lambda^{\prime}\left(\tau_{2}^{*}\right)\right\}}{\tau_{2}^{*} \omega_{2}^{*}} .
\end{gathered}
$$

By the results of Hassard et al. [26], the following results are obtained. 


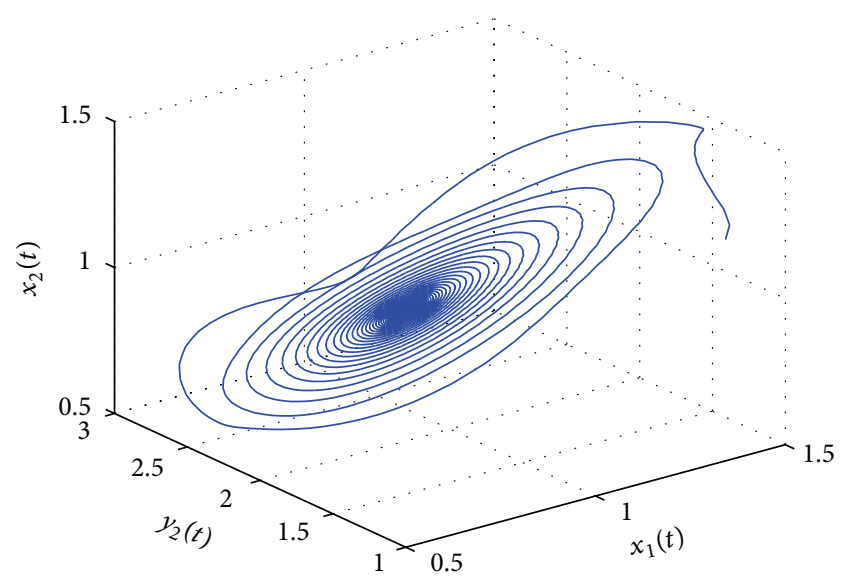

FIGURE 8: The phase plot of the states $x_{1}, x_{2}, y_{2}$ for $\tau_{2}=7.7500<$ $\tau_{20}=9.9183$.

Theorem 6. For system (5),

(i) the direction of the Hopf bifurcation is determined by $\mu_{2}$ : if $\mu_{2}>0$, the Hopf bifurcation is supercritical; if $\mu_{2}<0$, the Hopf bifurcation is subcritical;

(ii) the stability of the bifurcating periodic solutions is determined by $\beta_{2}$ : if $\beta_{2}<0$, the bifurcating periodic solutions are stable; if $\beta_{2}>0$, the bifurcating periodic solutions are unstable;

(iii) the period of the bifurcating periodic solution is determined by $T_{2}$ : if $T_{2}>0$, the period of the bifurcating periodic solutions increases; if $T_{2}<0$, the period of the bifurcating periodic solutions decreases.

\section{Numerical Simulation}

In this section, we present some numerical simulations to support the theoretical analysis in Sections 2 and 3. We consider the following system by taking the same coefficients as in [17]:

$$
\begin{gathered}
\frac{d x_{1}(t)}{d t}=5 x_{2}(t)-x_{1}(t) x_{1}\left(t-\tau_{1}\right)-2 x_{1}(t)-x_{1}(t) y_{2}(t) \\
\frac{d x_{2}(t)}{d t}=x_{1}(t)-x_{2}(t) \\
\frac{d y_{1}(t)}{d t}=2 x_{1}\left(t-\tau_{2}\right) y_{2}\left(t-\tau_{2}\right)-2.2 y_{1}(t) \\
\frac{d y_{2}(t)}{d t}=1.2 y_{1}(t)-y_{2}(t)
\end{gathered}
$$

from which we can get $a_{2} r r_{1} r_{2}=12, a d_{2} d_{4}\left(d_{3}+r_{2}\right)+$ $a_{2} d_{2} r_{2}\left(d_{1}+r_{1}\right)=7$. Obviously, $a_{2} r r_{1} r_{2}>a d_{2} d_{4}\left(d_{3}+\right.$ $\left.r_{2}\right)+a_{2} d_{2} r_{2}\left(d_{1}+r_{1}\right)$. Therefore, the condition $(H)$ is satisfied. Further, we get the unique positive equilibrium $E^{*}(0.9167,0.9167,1.7361,2.0833)$ of system (73) by MATLAB software package. Then, we have $A_{10}=4.5833, A_{11}=$ 7.5163, $A_{13} A_{12}=233.1899, A_{11} A_{12} A_{13}=1752.7$, and

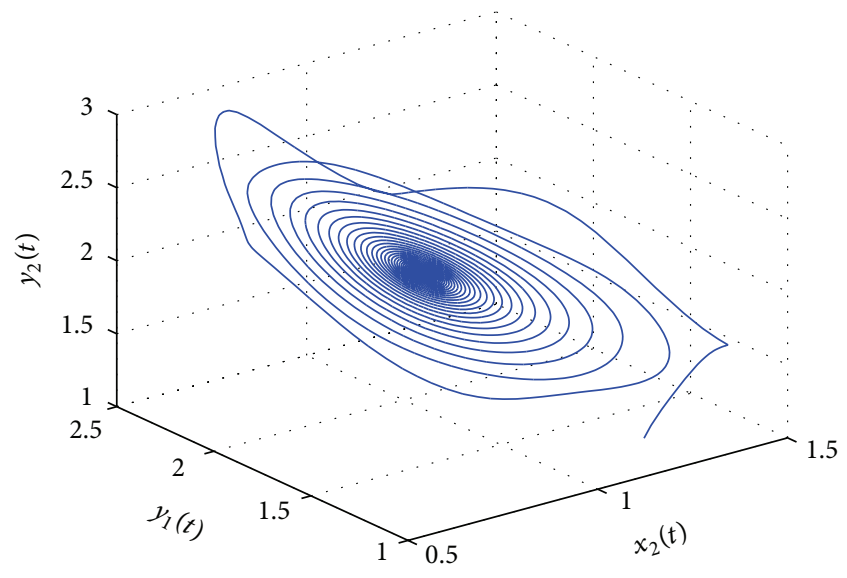

FIGURE 9: The phase plot of the states $x_{2}, y_{1}, y_{2}$ for $\tau_{2}=7.7500<$ $\tau_{20}=9.9183$.

$A_{11}^{2}+A_{10} A_{13}^{2}=525.5744$. Obviously, $A_{10}=4.5833>0$, $A_{13} A_{12}>A_{11}$, and $A_{11} A_{12} A_{13}>A_{11}^{2}+A_{10} A_{13}^{2}$. Namely, the conditions $\left(H_{11}\right)$ and $\left(H_{12}\right)$ hold.

For $\tau_{1}>0, \tau_{2}=0$. From (18), we have

$$
\omega_{1}^{8}+45.3999 \omega_{1}^{6}+284.0324 \omega_{1}^{4}-163.6041 \omega_{1}^{2}+21.0076=0 .
$$

Then, (20) becomes

$$
\begin{aligned}
v_{1}^{4}+45.3999 v_{1}^{3}+284.0324 v_{1}^{2} \\
-163.6041 v_{1}+21.0076=0
\end{aligned}
$$

and it has two positive real roots: $v_{11}=0.3397, v_{12}=$ 0.2003. Thus, the condition $\left(H_{21}\right)$ is satisfied. Further, we have $\omega_{10}=0.4475, \tau_{10}=4.5061$. Then, we get $f_{1}^{\prime}\left(v_{1 *}\right)=-44.3242 \neq 0$. Therefore, the condition $\left(H_{22}\right)$ holds. From Theorem 2, we know that the positive equilibrium $E^{*}(0.9167,0.9167,1.7361,2.0833)$ is asymptotically stable for $\tau_{1} \in[0,4.5061)$, which can be shown in Figures 1, 2, and 3. The positive equilibrium $E^{*}(0.9167,0.9167,1.7361,2.0833)$ is unstable when $\tau_{1}>$ 4.5061 and system (73) undergoes a Hopf bifurcation at $E^{*}(0.9167,0.9167,1.7361,2.0833)$, and a family of periodic solutions bifurcate from $E^{*}(0.9167,0.9167,1.7361,2.0833)$. This property can be illustrated by Figures 4-6. Similarly, we have $\omega_{20}=0.1517, \tau_{20}=9.9183$ for $\tau_{1}=0, \tau_{2}>$ 0 . For $\tau_{2}=7.7500 \in\left[0, \tau_{20}\right)$, the positive equilibrium $E^{*}(0.9167,0.9167,1.7361,2.0833)$ is asymptotically stable from Theorem 3 and this property can be shown in Figures 7,8 , and 9. If $\tau_{2}=10.7500>\tau_{20}=9.9183$, the positive equilibrium $E^{*}(0.9167,0.9167,1.7361,2.0833)$ is unstable and a Hopf bifurcation occurs, and the corresponding waveforms and phase plots are shown in Figures 10, 11, and 12.

For $\tau_{1}=\tau_{2}=\tau>0$, we can get $\omega_{0}=$ $0.3412, \tau_{0}=3.1043$. From Theorem 4, we know that the positive equilibrium $E^{*}(0.9167,0.9167,1.7361,2.0833)$ is asymptotically stable for $\tau \in\left[0, \tau_{0}\right)$, which can be illustrated by Figures 13, 14, and 15 . As can be seen from Figure 5 that when $\tau=2.7500 \in\left[0, \tau_{0}\right)$, the positive equilibrium 

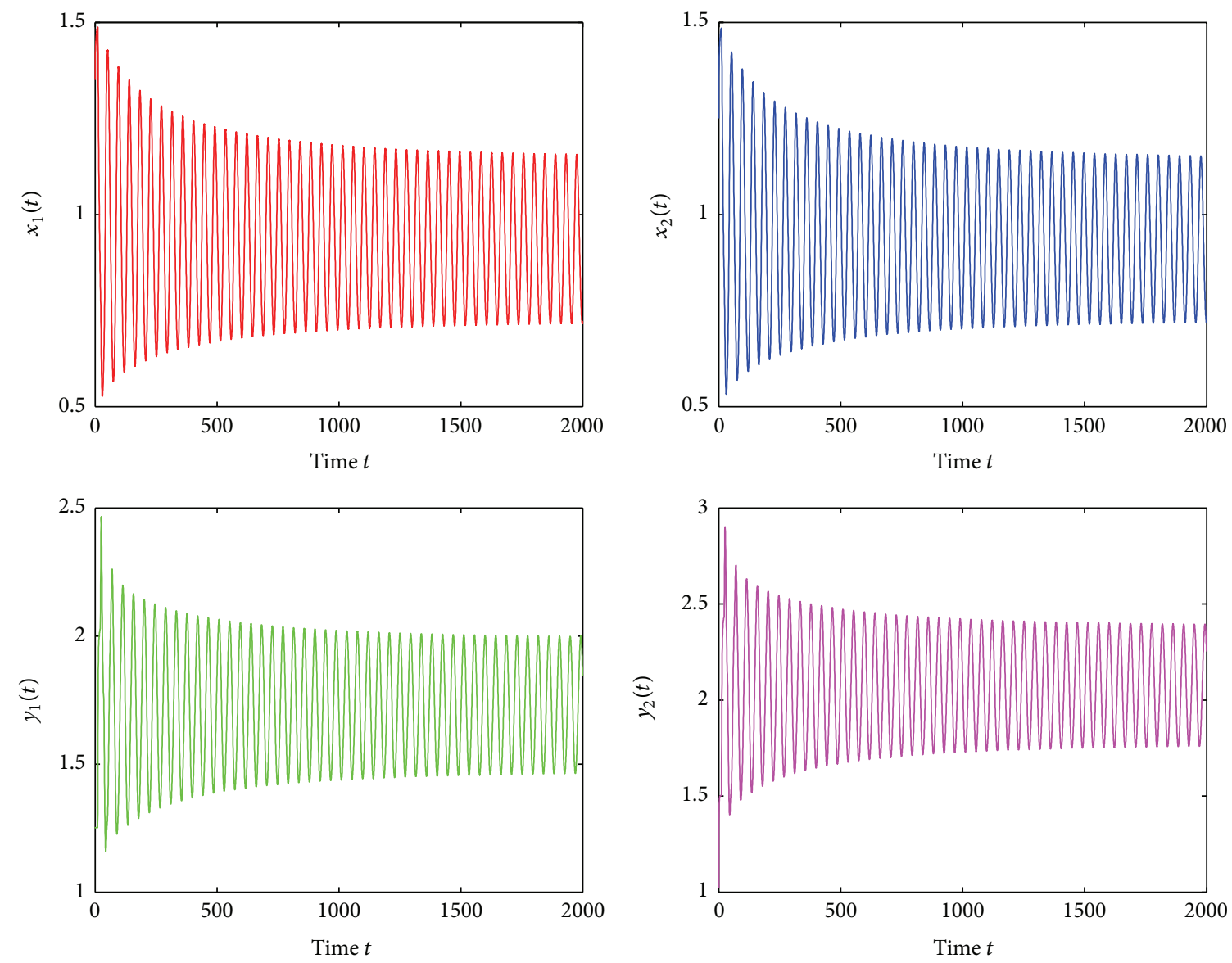

FIgURE 10: The track of the states $x_{1}, x_{2}, y_{1}, y_{2}$ for $\tau_{2}=10.7500>\tau_{20}=9.9183$.

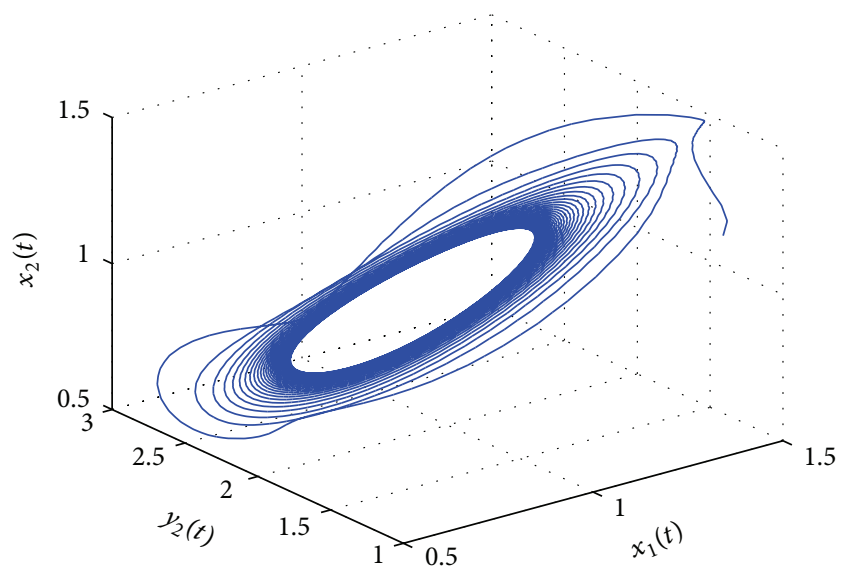

Figure 11: The phase plot of the states $x_{1}, x_{2}, y_{2}$ for $\tau_{2}=10.7500>$ $\tau_{20}=9.9183$.

$E^{*}(0.9167,0.9167,1.7361,2.0833)$ is asymptotically stable. However, if $\tau=3.2500>\tau_{0}=3.1043$, then the positive equilibrium $E^{*}(0.9167,0.9167,1.7361,2.0833)$ becomes unstable and a family of bifurcating periodic solutions occur, which is illustrated by Figures 16, 17, and 18.

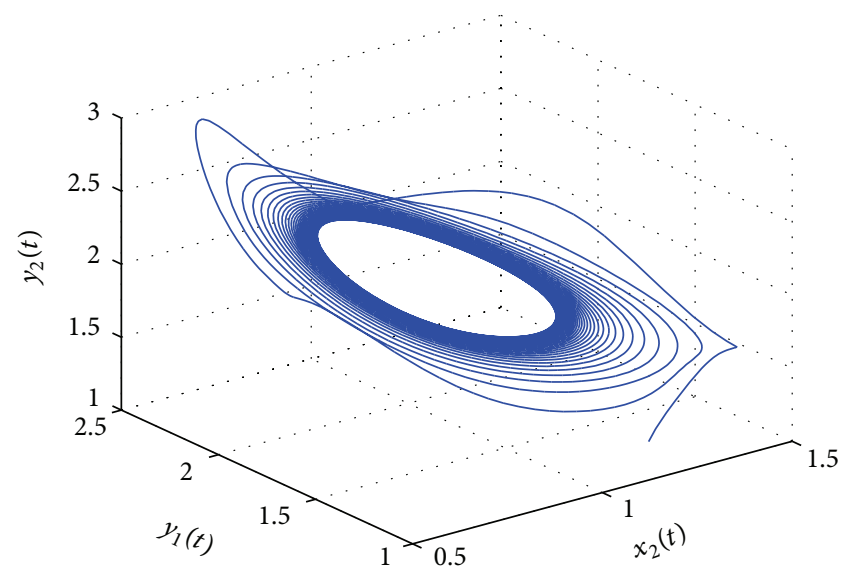

FIGURE 12: The phase plot of the states $x_{2}, y_{1}, y_{2}$ for $\tau_{2}=10.7500>$ $\tau_{20}=9.9183$.

Lastly, regarding $\tau_{2}$ as a parameter and letting $\tau_{1}=$ $2.5 \in\left(0, \tau_{10}\right)$, we can obtain that $\omega_{2}^{*}=0.2253$. Further, we have $\tau_{2}^{*}=6.6494$ and $\lambda^{\prime}\left(\tau_{2}^{*}\right)=0.0466+0.0314 i$. Letting $\tau_{2}=5.5500 \in\left[0, \tau_{1}^{*}\right)$, we can know that the positive equilibrium $E^{*}(0.9167,0.9167,1.7361,2.0833)$ is asymptotically stable from Theorem 5. When $\tau_{2}=$ 

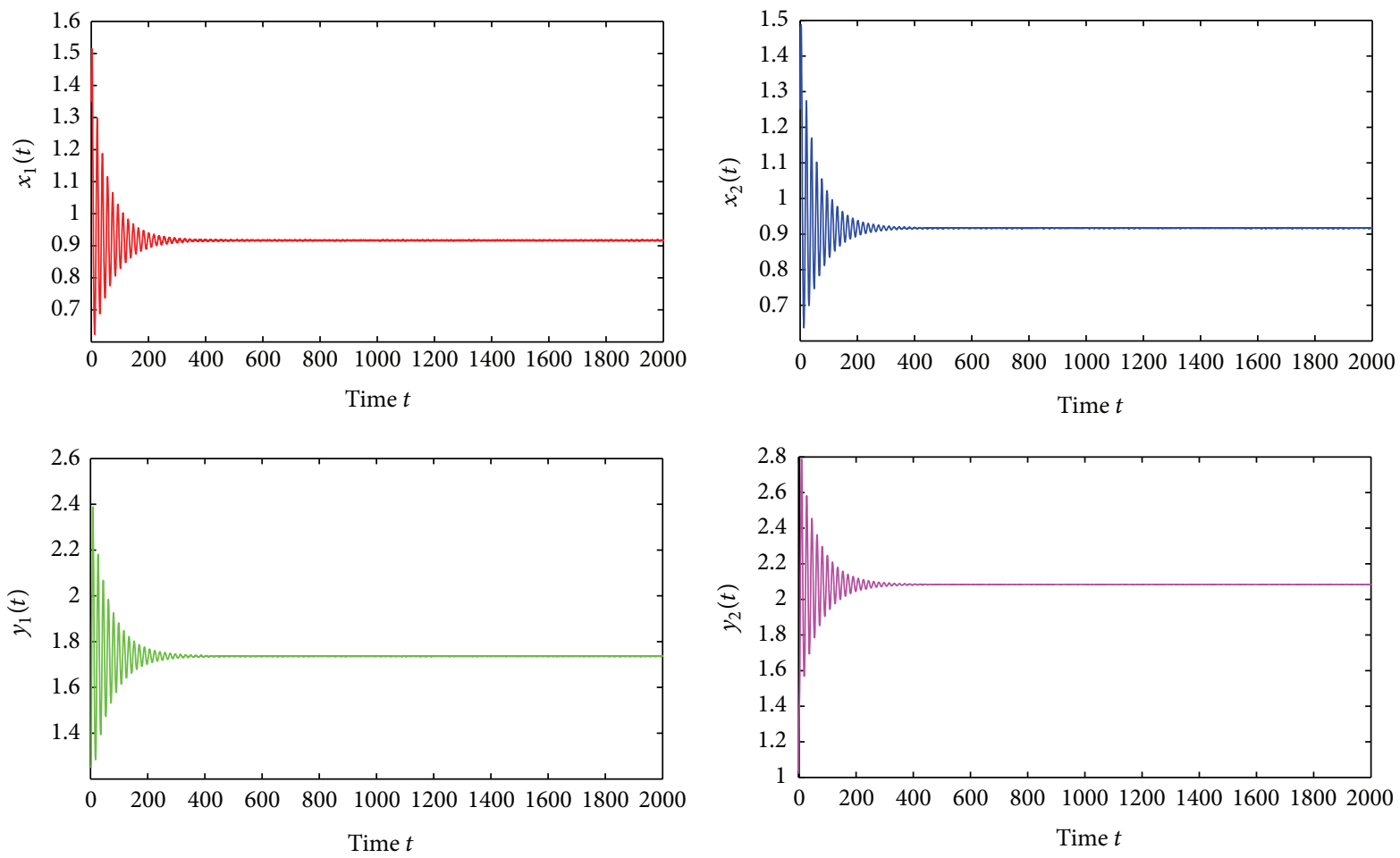

FIgURE 13: The track of the states $x_{1}, x_{2}, y_{1}, y_{2}$ for $\tau=2.7500<\tau_{0}=3.1043$.

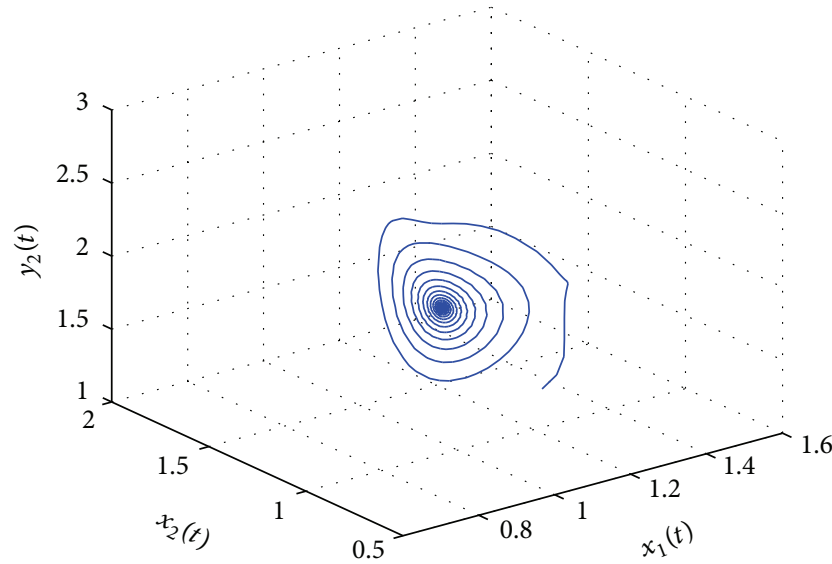

FIGURE 14: The phase plot of the states $x_{1}, x_{2}, y_{2}$ for $\tau=2.7500<$ $\tau_{0}=3.1043$.

$7.5000>\tau_{2}^{*}=6.6494$, then the positive equilibrium $E^{*}(0.9167,0.9167,1.7361,2.0833)$ becomes unstable and a Hopf bifurcation occurs. The corresponding waveforms and phase plots are illustrated in Figures 19, 20, 21, 22, 23, and 24.

In addition, for system (73), we have $C_{1}(0)=-31.3276+$ $4.4079 i$ by a simple computation. Further, from (72), we have $\mu_{2}=672.2661>0, \beta_{2}=-62.6552<0$, and $T_{2}=$ $-17.0330<0$. Therefore, by Theorem 6 , we can know that the Hopf bifurcation is supercritical, the bifurcating periodic solutions are stable, and the period of the bifurcated periodic solutions decrease. Since the bifurcating periodic solutions of

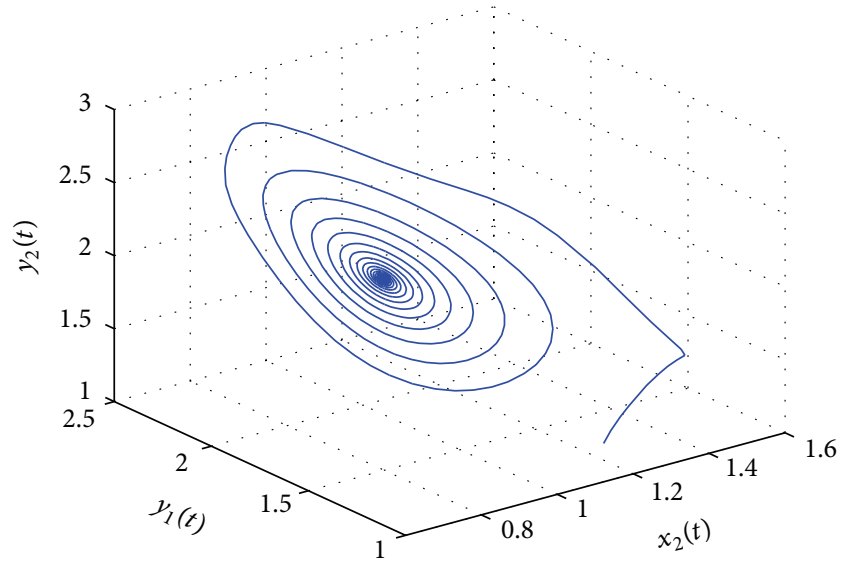

Figure 15: The phase plot of the states $x_{2}, y_{1}, y_{2}$ for $\tau=2.7500<$ $\tau_{0}=3.1043$.

system (73) are stable, the predator and the prey species in system (73) can coexist in an oscillatory mode from the view of biology.

\section{Conclusions}

In this paper, a delayed predator-prey system with stagestructure for both the predator and the prey population is investigated. The sufficient conditions for the local stability of the positive equilibrium and the existence of Hopf bifurcation for the possible combinations of two delays are obtained. 

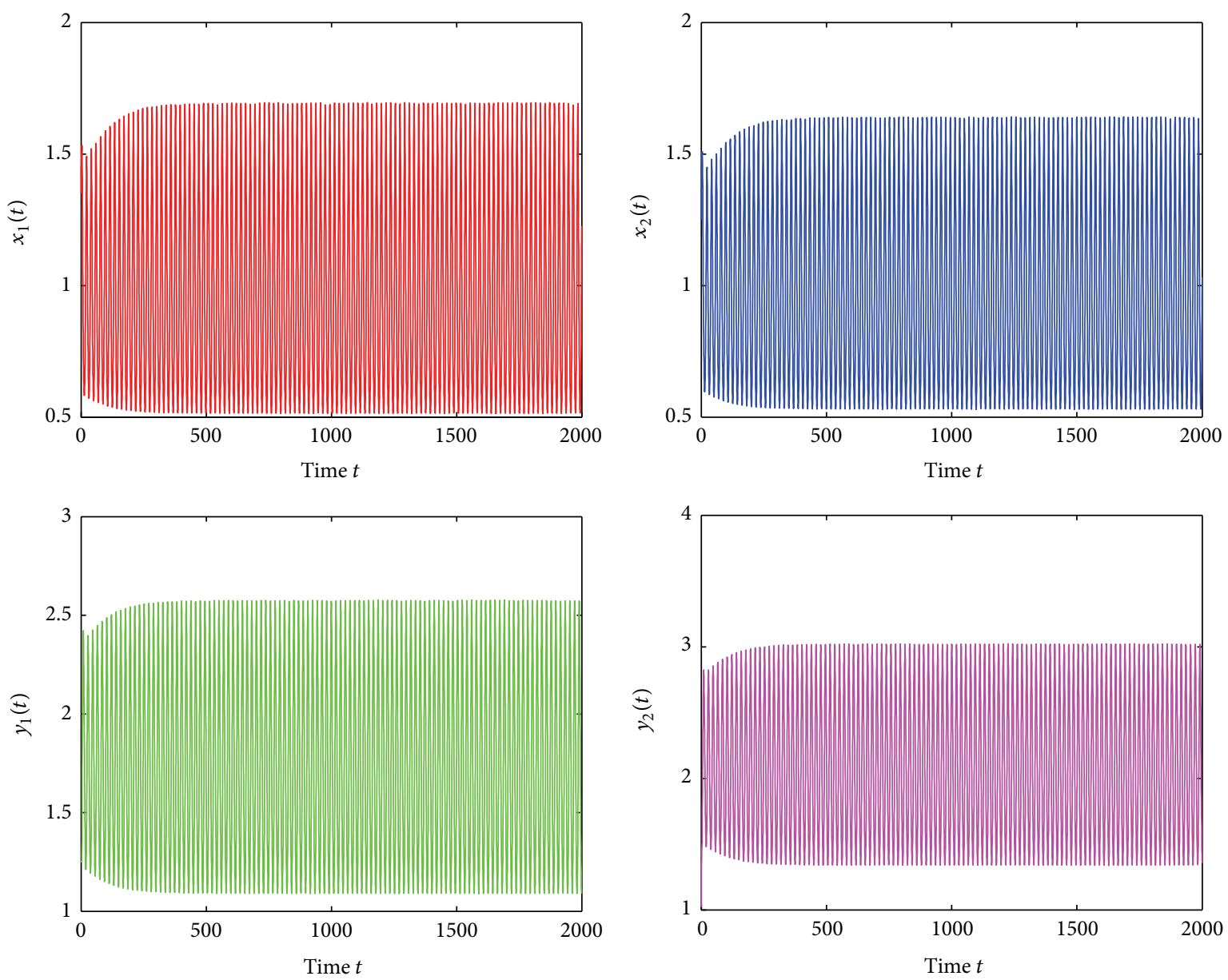

FIGURE 16: The track of the states $x_{1}, x_{2}, y_{1}, y_{2}$ for $\tau=3.2500>\tau_{0}=3.1043$.

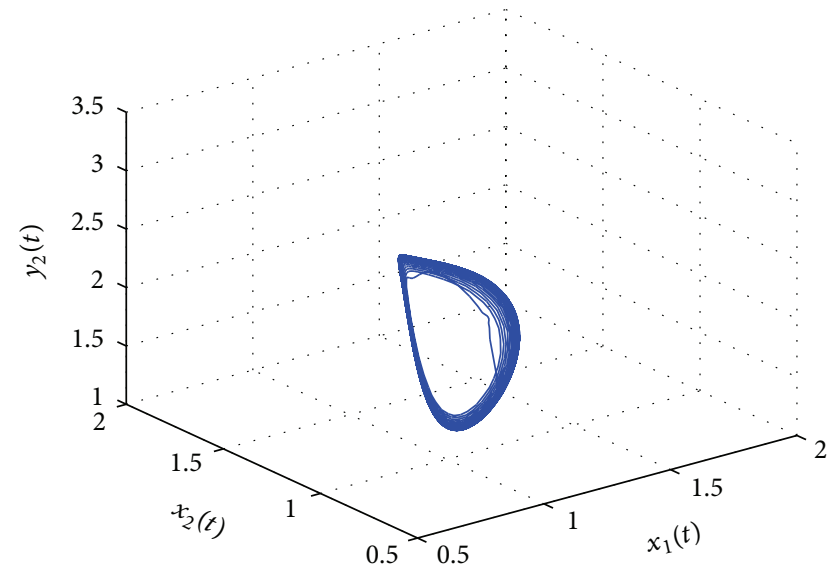

Figure 17: The phase plot of the states $x_{1}, x_{2}, y_{2}$ for $\tau=3.2500>$ $\tau_{0}=3.1043$.

The main results are given in Theorems $2-5$. The results show that the two time delays can play a complicated role in system (5). Furthermore, the explicit formulae determining the direction of the bifurcation and the stability of the bifurcating periodic solutions are established when $\tau_{2}>0$

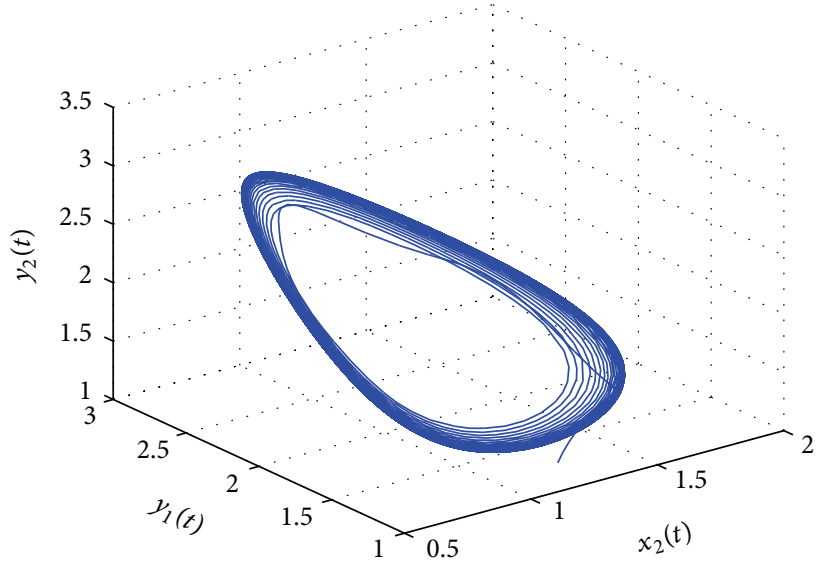

FIGURE 18: The phase plot of the states $x_{2}, y_{1}, y_{2}$ for $\tau=3.2500>$ $\tau_{0}=3.1043$.

and $\tau_{1} \in\left(0, \tau_{10}\right)$ by using the normal form theory and center manifold theorem. The main results are given in Theorem 6 . If the bifurcating periodic solutions are stable, the predator and the prey species can coexist in an oscillatory mode from the view of biology. Compared with literature [17], we 

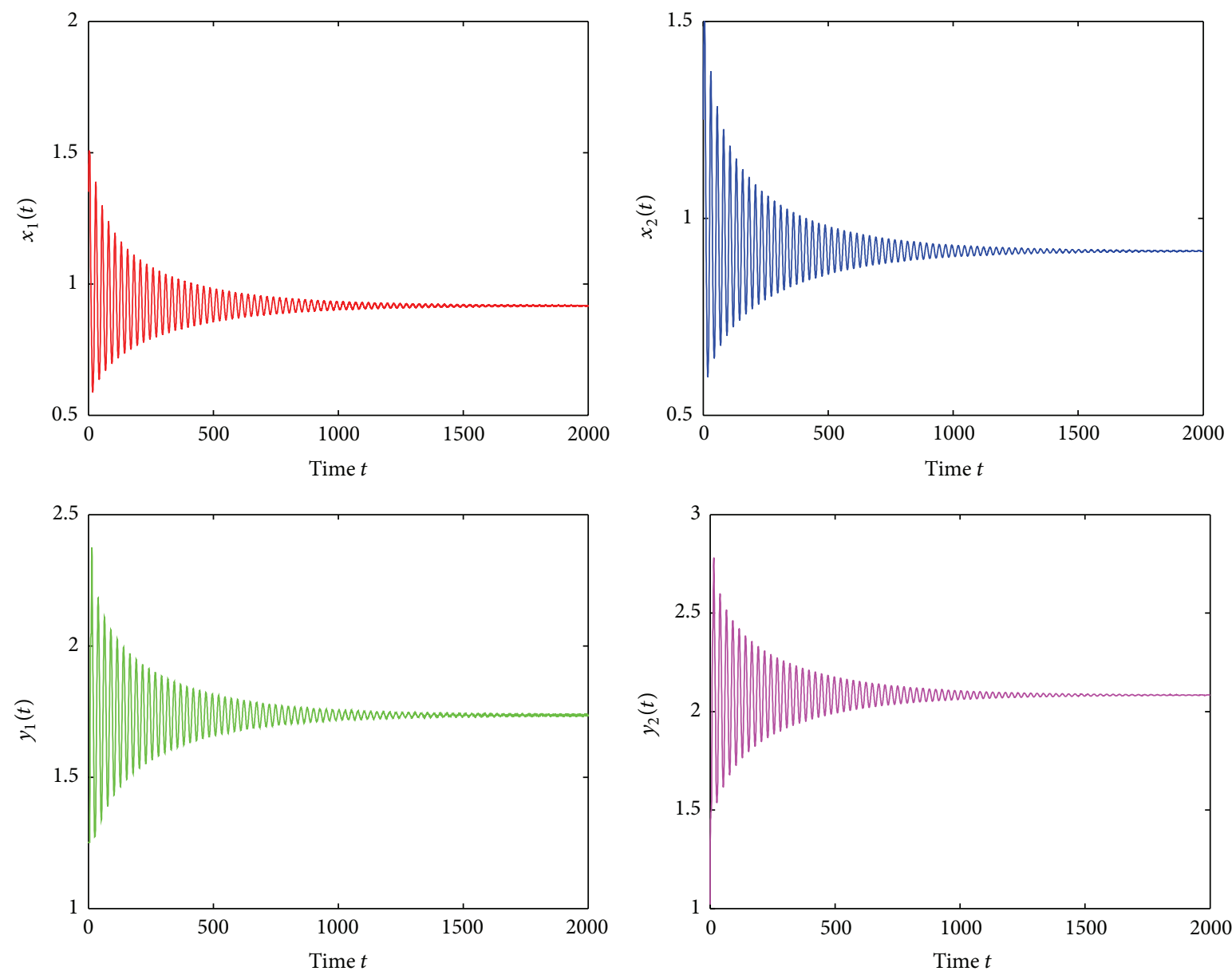

Figure 19: The track of the states $x_{1}, x_{2}, y_{1}, y_{2}$ for $\tau_{2}=5.5500<\tau_{2}^{*}=6.6494$ and $\tau_{1}^{*}=2.5000$.

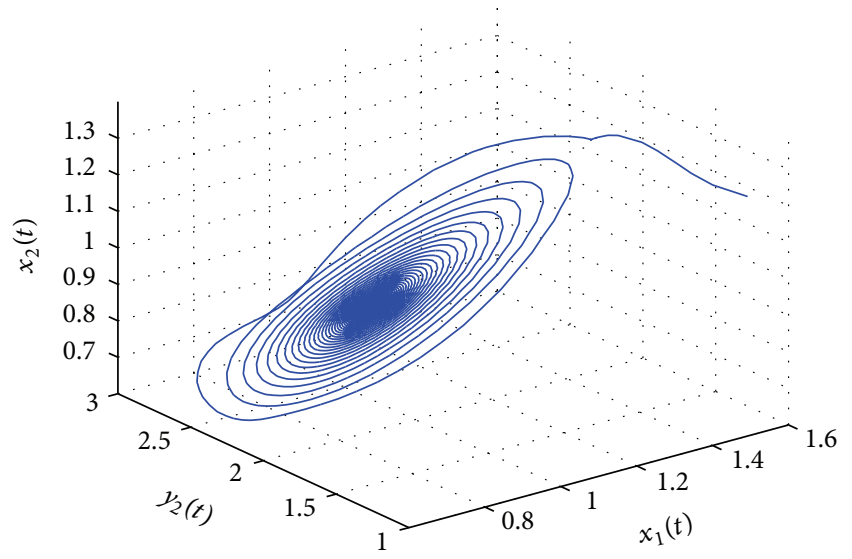

Figure 20: The phase plot of the states $x_{1}, x_{2}, y_{2}$ for $\tau_{2}=5.5500<$ $\tau_{2}^{*}=6.6494$ and $\tau_{1}^{*}=2.5000$.

consider not only the time delay due to the gestation of the mature predator but also the negative feedback delay of the immature prey. Wang [17] obtained that the species in system (5) with only the time delay due to the gestation of the mature predator could coexist under some conditions. However, we

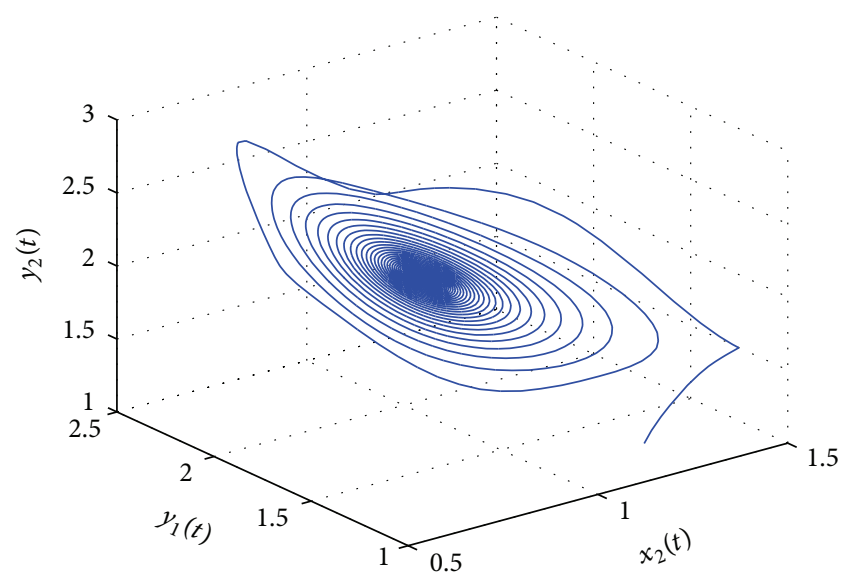

Figure 21: The phase plot of the states $x_{2}, y_{1}, y_{2}$ for $\tau_{2}=5.5500<$ $\tau_{2}^{*}=6.6494$ and $\tau_{1}^{*}=2.5000$.

get that the species could also coexist with some available time delays of the immature prey and the mature predator under certain conditions. This is valuable from the view of ecology. Some numerical simulations are also included to support the obtained theoretical results. 

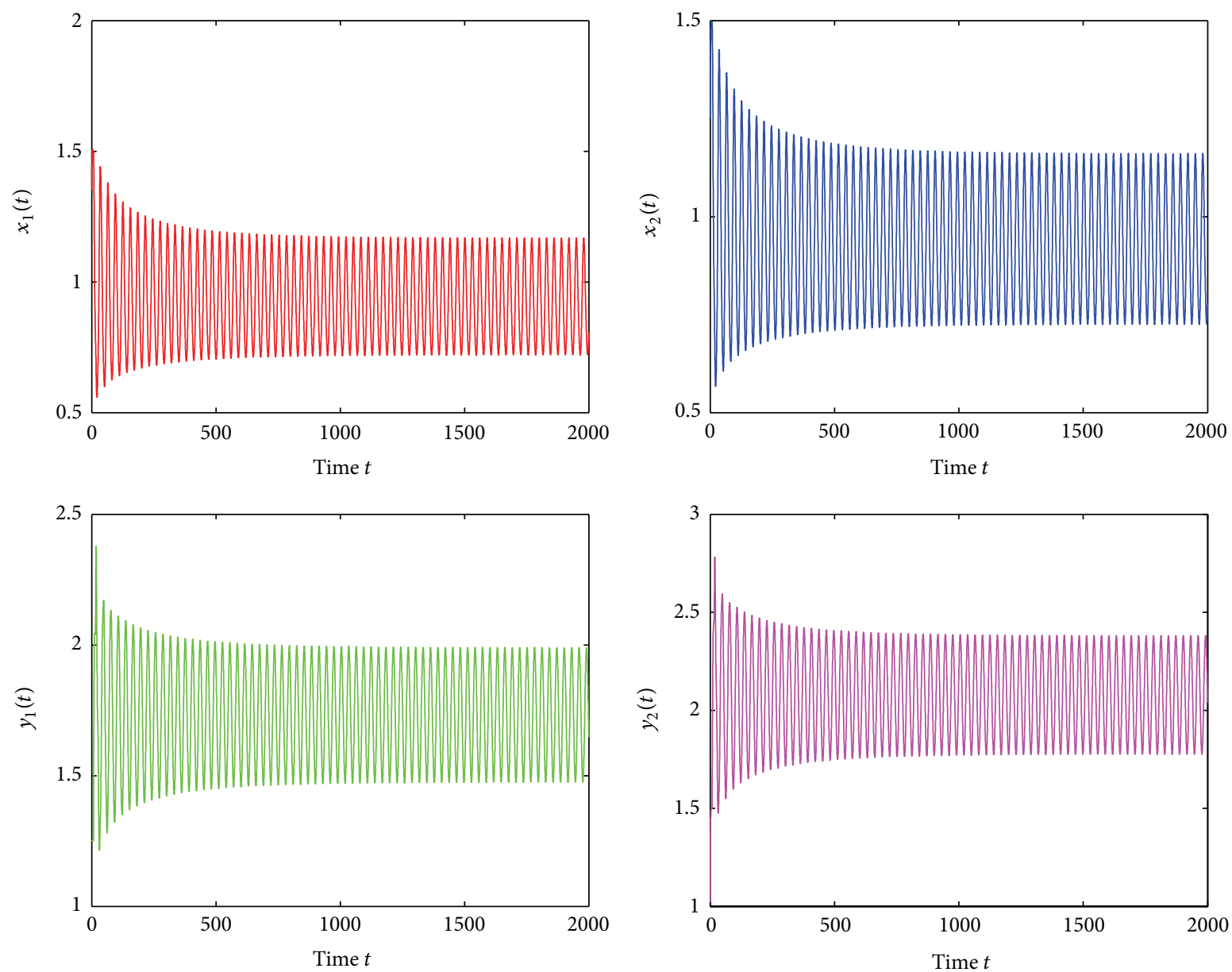

Figure 22: The track of the states $x_{1}, x_{2}, y_{1}, y_{2}$ for $\tau_{2}=7.5000>\tau_{2}^{*}=6.6494$ and $\tau_{1}^{*}=2.5000$.

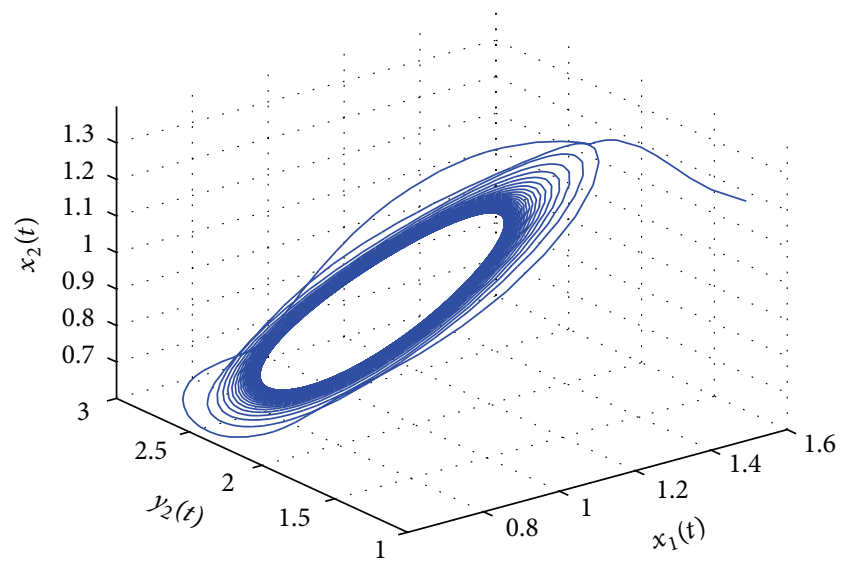

FIGURE 23: The phase plot of the states $x_{1}, x_{2}, y_{2}$ for $\tau_{2}=7.5000>$ $\tau_{2}^{*}=6.6494$ and $\tau_{1}^{*}=2.5000$.

\section{Conflict of Interests}

The authors declare that there is no conflict of interests regarding the publication of this article.

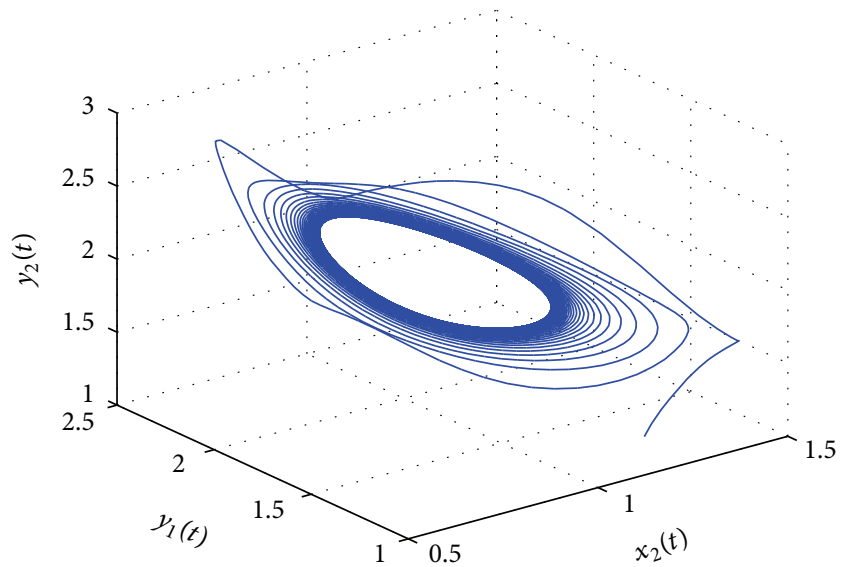

FIGURE 24: The phase plot of the states $x_{2}, y_{1}, y_{2}$ for $\tau_{2}=7.5000>$ $\tau_{2}^{*}=6.6494$ and $\tau_{1}^{*}=2.5000$.

\section{Acknowledgments}

This work was supported by the National Nature Science Foundation of China (61273070), a project funded by the Priority Academic Program Development of Jiangsu Higher 
Education Institutions and Natural Science Foundation of the Higher Education Institutions of Anhui Province (KJ2013A003, KJ2013B137).

\section{References}

[1] F. Brauer and M. Zhien, "Stability of stage-structured population models," Journal of Mathematical Analysis and Applications, vol. 126, no. 2, pp. 301-315, 1987.

[2] W. G. Aiello and H. I. Freedman, "A time-delay model of singlespecies growth with stage structure," Mathematical Biosciences, vol. 101, no. 2, pp. 139-153, 1990.

[3] H. I. Freedman and J. Wu, "Persistence and global asymptotic stability of single species dispersal models with stage structure," Quarterly of Applied Mathematics, vol. 49, pp. 351-371, 1991.

[4] W. G. Aiello, H. I. Freedman, and J. Wu, "Analysis of a model representing stage-structured population growth with statedependent time delay," SIAM Journal on Applied Mathematics, vol. 52, pp. 855-869, 1992.

[5] Y. Kuang and J. So, "Analysis of a delayed two-stage population model with space-limited recruitment," SIAM Journal on Applied Mathematics, vol. 55, no. 6, pp. 1675-1696, 1995.

[6] Z. Zhang and Z. Hou, "Existence of four positive periodic solutions for a ratio-dependent predator-prey system with multiple exploited (or harvesting) terms," Nonlinear Analysis: Real World Applications, vol. 11, no. 3, pp. 1560-1571, 2010.

[7] R. Xu, M. A. J. Chaplain, and F. A. Davidson, "Periodic solutions of a predator-prey model with stage structure for predator," Applied Mathematics and Computation, vol. 154, no. 3, pp. 847870, 2004.

[8] Z. Zhang and J. Luo, "Multiple periodic solutions of a delayed predatorprey system with stage structure for the predator," Nonlinear Analysis: Real World Applications, vol. 11, no. 5, pp. 4109-4120, 2010

[9] Y. Qu and J. Wei, "Bifurcation analysis in a time-delay model for prey-predator growth with stage-structure," Nonlinear Dynamics, vol. 49, no. 1-2, pp. 285-294, 2007.

[10] L. Cai, J. Yu, and G. Zhu, "A stage-structured predator-prey model with Beddington-DeAngelis functional response," Journal of Applied Mathematics and Computing, vol. 26, no. 1-2, pp. 85-103, 2008.

[11] Z. Zhang and L. Wang, "Global attractivity of a positive periodic solution for a nonautonomous stage structured population dynamics with time delay and diffusion," Journal of Mathematical Analysis and Applications, vol. 319, no. 1, pp. 17-33, 2006.

[12] R. Xu, "Global dynamics of a predatorprey model with time delay and stage structure for the prey," Nonlinear Analysis: Real World Applications, vol. 12, no. 4, pp. 2151-2162, 2011.

[13] R. Xu, "Global stability and Hopf bifurcation of a predatorprey model with stage structure and delayed predator response," Nonlinear Dynamics, vol. 67, no. 2, pp. 1683-1693, 2012.

[14] X.-K. Sun, H.-F. Huo, and H. Xiang, "Bifurcation and stability analysis in predator-prey model with a stage-structure for predator," Nonlinear Dynamics, vol. 58, no. 3, pp. 497-513, 2009.

[15] F. Li and H. Li, "Hopf bifurcation of a predator-prey model with time delay and stage structure for the prey," Mathematical and Computer Modelling, vol. 55, no. 3-4, pp. 672-679, 2012.

[16] F. D. Chen, W. L. Chen, Y. M. Wu, and Z. Z. Ma, "Permanence of a stage-structured predator-prey system," Applied Mathematics and Computation, vol. 219, pp. 8856-8862, 2013.
[17] L. S. Wang, "Stability and hopf bifurcation in a predatorprey model with stage structure and time delay," Mathematica Applicata, vol. 25, pp. 131-139, 2012.

[18] Y. Song, M. Han, and Y. Peng, "Stability and Hopf bifurcations in a competitive Lotka-Volterra system with two delays," Chaos, Solitons and Fractals, vol. 22, no. 5, pp. 1139-1148, 2004.

[19] X.-Y. Meng, H.-F. Huo, and H. Xiang, "Hopf bifurcation in a three-species system with delays," Journal of Applied Mathematics and Computing, vol. 35, no. 1-2, pp. 635-661, 2011.

[20] G.-H. Cui and X.-P. Yan, "Stability and bifurcation analysis on a three-species food chain system with two delays," Communications in Nonlinear Science and Numerical Simulation, vol. 16, no. 9, pp. 3704-3720, 2011.

[21] C. Xu, X. Tang, M. Liao, and X. He, "Bifurcation analysis in a delayed Lokta-Volterra predator-prey model with two delays," Nonlinear Dynamics, vol. 66, no. 1-2, pp. 169-183, 2011.

[22] G.-P. Hu, W.-T. Li, and X.-P. Yan, "Hopf bifurcations in a predator-prey system with multiple delays," Chaos, Solitons and Fractals, vol. 42, no. 2, pp. 1273-1285, 2009.

[23] S. Gakkhar and A. Singh, "Complex dynamics in a prey predator system with multiple delays," Communications in Nonlinear Science and Numerical Simulation, vol. 17, no. 2, pp. 914-929, 2012.

[24] M. Liao, X. Tang, and C. Xu, "Bifurcation analysis for a threespecies predator-prey system with two delays," Communications in Nonlinear Science and Numerical Simulation, vol. 17, no. 1, pp. 183-194, 2012.

[25] T. Zhang, J. Liu, and Z. Teng, "Stability of Hopf bifurcation of a delayed SIRS epidemic model with stage structure," Nonlinear Analysis: Real World Applications, vol. 11, no. 1, pp. 293-306, 2010.

[26] B. D. Hassard, N. D. Kazarinoff, and Y. H. Wan, Theory and Applications of Hopf Bifurcation, Cambridge University Press, Cambridge, UK, 1981. 


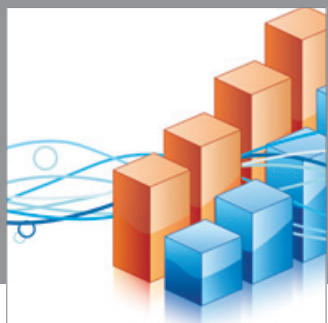

Advances in

Operations Research

mansans

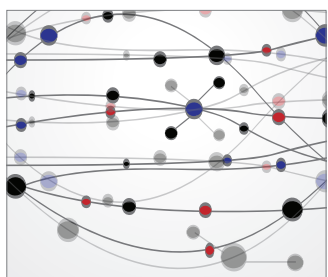

The Scientific World Journal
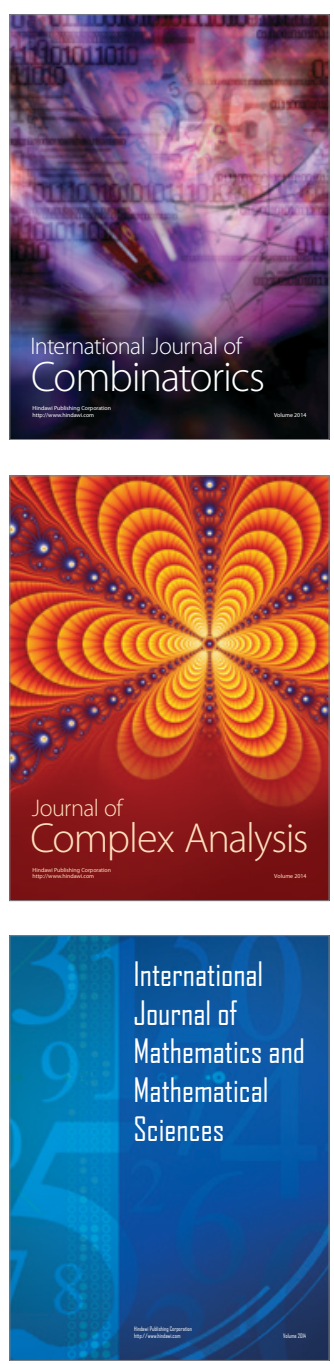
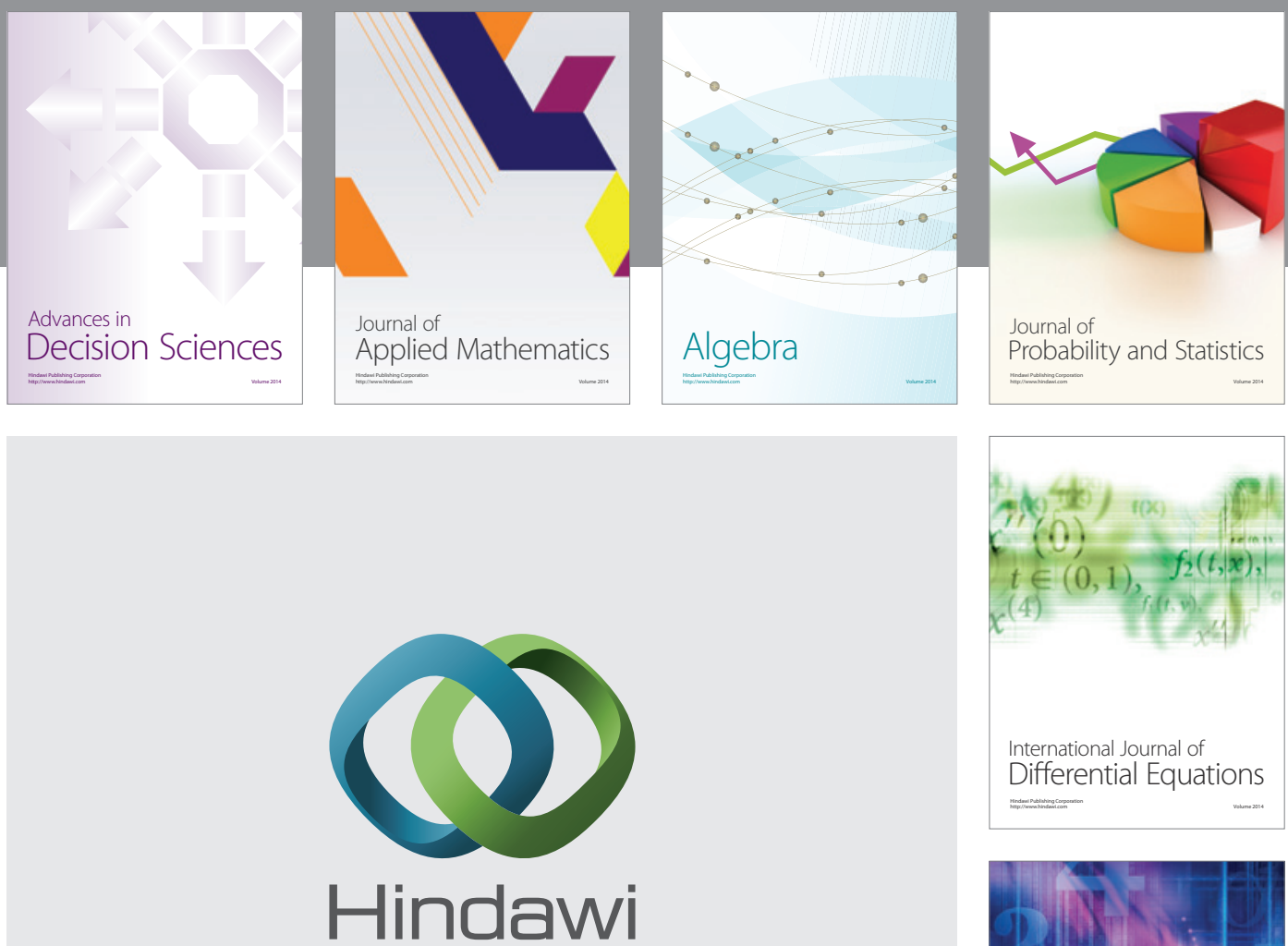

Submit your manuscripts at http://www.hindawi.com
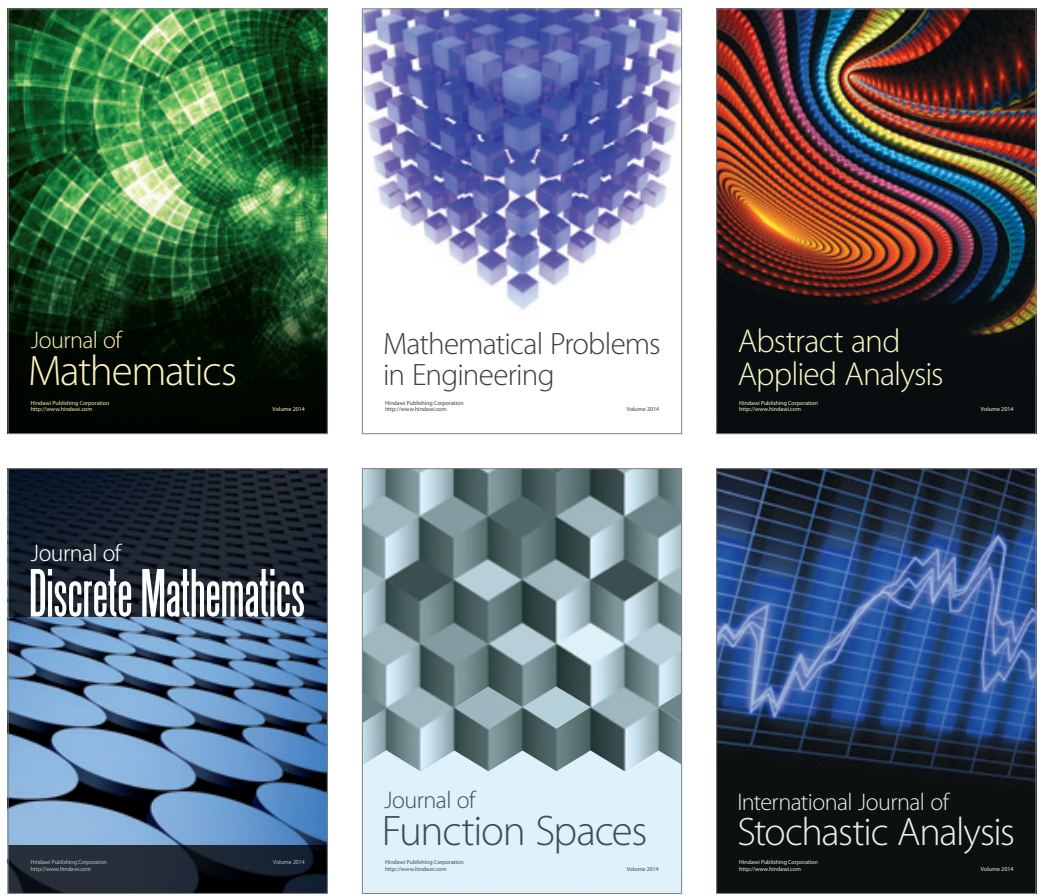

Journal of

Function Spaces

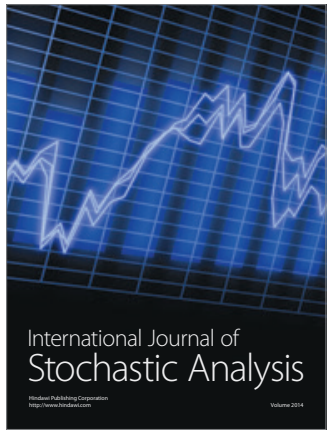

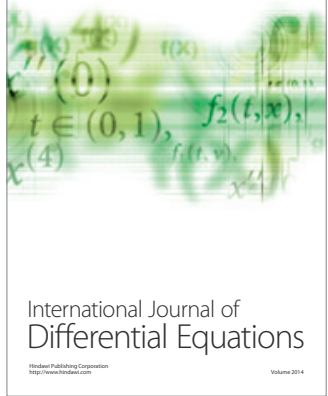
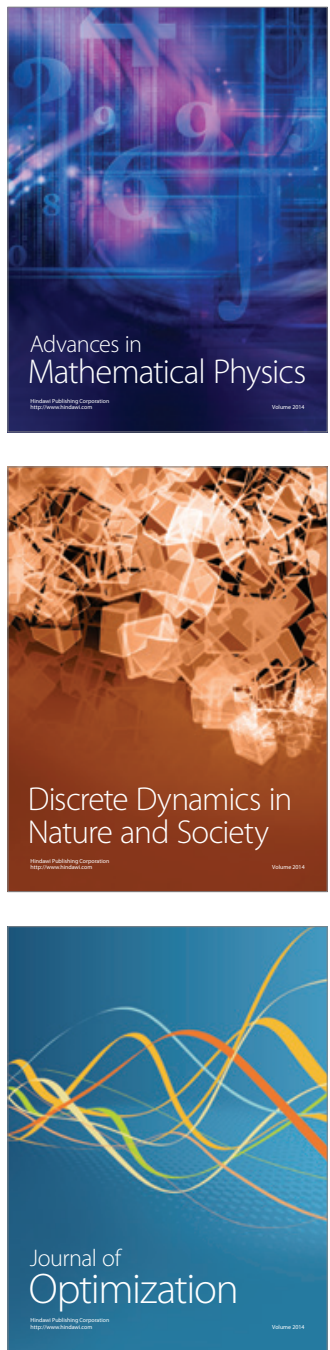